\title{
CONTATTO LINGUISTICO E PERCEZIONE. PER UNA VALUTAZIONE DELLE VOCI D'ORIGINE SARDA IN TABARCHINO*
}

Questo saggio intende approfondire il tema del contatto linguistico e dei meccanismi percettivi connessi, esaminando criticamente il problema dell 'interferenza sarda nella parlata ligure-tabarchina di Carloforte e Calasetta. Malgrado l'esistenza di studi specifici su questa varietà, una valutazione complessiva della componente lessicale sarda nelle sue motivazioni storico-linguistiche e sociolinguistiche non è mai stata avanzata; nondimeno, essa assume particolare interesse per quanto attiene non solo ai meccanismi di assunzione dei prestiti, ma anche alla percezione dei fenomeni di contatto da parte dei parlanti.

1. «Però, anche riuscendo a mettere insieme parecchie centinaia di voci accattate dal sardo, non si riuscirebbe per questo a dimostrare che il tabarchino non è più un dialetto genovese»: l'affermazione di Gino Bottiglioni, quasi a conclusione del primo saggio scientifico dedicato alla parlata ligure di Sardegna ${ }^{1}$ può apparire scontata dal momento in cui, nella descrizione tipologica di un idioma, viene generalmente attribuita, piuttosto che al lessico, un'importanza determinante ai fattori strutturali, fonetici e morfosintattici. Per di più, la preponderanza dell'eredità genovese nella parlata tabarchina è tale ${ }^{2}$, da impedire comunque una diversa ipotesi classificatoria ${ }^{3}$.

Alberto Sobrero, in un suo saggio più volte pubblicato ${ }^{4}$, aveva individuato proprio in una progressiva «penetrazione del tipo sardo» ${ }^{5}$ la manifestazione di una «fase preagonica del dialetto» ${ }^{6}$ : tale previsione, a distanza di tre decenni, pare oggi tuttaltro che confermata alla luce della singolare tenuta della fedeltà linguistica dei parlanti ${ }^{7}$.

2. L'ipotesi del Sobrero era basata tra l'altro su una valutazione diacronica dell'apporto lessicale sardo fondata su opinioni largamente accreditate relative alla realtà socioeconomica tabarchina: Carloforte e Calasetta «paesi di mare» ${ }^{8}$ sostanzialmente estranei alle pratiche agricole, avrebbero assunto solo alcuni sardismi di ambito tecnico-rurale che «potrebbero non essere così antichi come appare a prima vista» ${ }^{9}$, e ulteriormente incrementati, durante la recente fase di disgregazione, da un apporto in grado di «aumentare enormemente le interferenze» ${ }^{10} \mathrm{e}$ di appoggiare quindi i fenomeni di interferenza che Sobrero riscontrava a livello strutturale.

Tuttavia, non soltanto la struttura socio-economica di Carloforte e Calasetta non pare giustificare una simile lettura del fenomeno di prestito, ma neppure la prospettiva diacronica, alla luce di una conoscenza più approfondita delle vicende storiche dei

* Ringrazio per il sostegno e per i preziosi consigli la prof.ssa Antonietta DETTORI e il prof. Vincenzo ORIOLES. 
Tabarchini, consente di attribuire all'elemento sardo un carattere così recente e un effetto così dirompente nella realtà linguistica e sociolinguistica delle due comunità.

Una visione meno antagonistica dei rapporti di forza tra l'adstrato sardo e l'isola linguistica ligure consente forse di rivalutare, oggi, l'apporto di prestito come elemento costitutivo della specificità tabarchina - rispetto alla madrepatria genovese, certo, ma anche al contesto isolano -, e di verificarne la funzione come elemento di vitalità e di tenuta all'interno di un sistema che si rivela in grado di assorbire elementi lessicali strutturalmente estranei, e di arricchirsi mediante apporti allogeni anche consistenti, senza con ciò abdicare a un'originalità tipologica che rappresenta l'elemento certamente più vistoso di una specificità, che non si limita, peraltro, al solo dato linguistico ${ }^{11}$.

Alla valutazione di Bottiglioni dà credito il più recente studio di Eduardo Blasco Ferrer ${ }^{12}$, che, recuperando e aggiornando la prospettiva classificatoria ripudiata da Sobrero, dimostra l'ineludibilità del dato comparativo anche a livello di valutazione sociolinguistica ${ }^{13}$.

3. Per una corretta interpretazione dell'apporto lessicale sardo in tabarchino, va chiarito innanzitutto che per certi aspetti, e malgrado il forte radicamento delle due comunità, Carloforte e Calasetta ebbero sempre la connotazione di insediamenti coloniali, in un rapporto col retroterra basato principalmente sullo sfruttamento delle risorse, $\mathrm{e}$, almeno in parte, della manodopera indigena: attraverso l'intermediazione nella distribuzione dei prodotti di tonnara, ad esempio, o del minerale sulcitano, ma anche mediante il ricorso a giornalieri e stagionali sardi nella coltivazione intensiva della vigna $\mathrm{e}$ in altre pratiche agricole, soprattutto a Calasetta.

L'esclusività dei rapporti con gli ambienti economici e mercantili genovesi implicava inoltre l'assimilazione linguistica di quanti -- numerosi soprattutto nell'Ottocento - si trasferivano a Carloforte e Calasetta dalla stessa Sardegna, dalle regioni dell'Italia meridionale o da altri paesi ${ }^{14}$.

Queste caratteristiche «coloniali» dell'esperienza tabarchina si protraggono a lungo, dal momento della fondazione di Carloforte e Calasetta nel sec. XVIII ${ }^{15}$, in virtù di modelli di specializzazione economica sempre e comunque basati sul raccordo con una rete di distribuzione e di intermediazione sostanzialmente estranea al contesto sardo e facente capo per lo più a Genova e alla Liguria ${ }^{16}$ : la vitalità - anche attuale - del dialetto non soddisfa quindi esigenze meramente legate a un fattore distintivo e connotante rispetto al retroterra sardo, ma presenta, in diacronia, ben più consistenti motivazioni di ordine pratico: esse spiegano, del resto, il costante aggiornamento del tabarchino rispetto alle innovazioni prodottesi nel modello urbano genovese fino a tempi recenti ${ }^{17}$.

Salvare l'intercomprensione rispetto alla parlata della madrepatria, espungendo progressivamente i caratteri «rurali» della parlata originaria e limitando a campi semantici specifici l'apporto lessicale esogeno (non soltanto quello sardo), rappresentò l'aspetto vincente della strategia linguistica delle comunità tabarchine ${ }^{18}$, aspetto sul quale si sovrappose anche, com'è naturale, una prospettiva legata a fattori di (auto)ri- 
conoscimento collettivo e a una presa di distanza dal contesto sardo, sempre legata tuttavia a motivi di specializzazione economica prima che alla strutturazione/invenzione di un'alterità etnica.

4. Proprio la prospettiva «coloniale» dell'esperienza tabarchina favorì dunque un'assunzione precoce di voci sarde, limitate per lo più ad ambiti semantici che non interferissero con la funzionalità del sistema rispetto alle consuetudini linguistiche dei centri in rapporto ai quali si giustificava il mantenimento (e la promozione) della specificità linguistica. Secondo modalità ben note in altre realtà coloniali, il sardo penetrò facilmente, allora - e precocemente - nella terminologia legata alla descrizione dell'ambiente (a cominciare dal mantenimento parziale della toponomastica originaria) ${ }^{19}$, ai sistemi di produzione, ai rapporti con le istituzioni e i mercati locali, all'adozione di determinate consuetudini alimentari e domestiche conseguenti all'impianto delle comunità in una realtà profondamente diversa da quella originaria; ma interferì assai poco nel lessico di base: i prestiti si connotarono essenzialmente come tecnicismi, per i quali, in molti casi, non si presentò neppure l'esigenza di un' assimilazione alle strutture fonetiche e morfologiche del genovese ${ }^{20}$; in altri casi invece, il rivestimento potrebbe forse riflettere una maggiore antichità del prestito ${ }^{21}$.

Che l'assunzione relativamente consistente di voci sarde sia piuttosto antica, lo dimostrano del resto anche la documentazione storica e alcuni aspetti conservativi della fonetica di questo lessico rispetto all'evoluzione della varietà sarda sulcitana.

Il termine muštazáfu 'guardia civica' è presente, ad esempio, nei verbali dei primi consigli comunali di Carloforte ${ }^{22}$, e paiono sarde due denominazioni locali di una pianta medicinale (Solanum nigrum) citata dall'Angius nel suo contributo per il Dizionario del Casalis: minca de lù per Carloforte e scala-bàxiu per Calasetta, voce tuttora in uso ${ }^{23}$.

Voci come kartálu 'tipo di cesta' o šivẹla 'catino di terracotta' riflettono un adeguamento automatico $-d d->-l$ - successivamente sostituito, in altri prestiti, dalla mera equivalenza - $d d->-d$ - mentre il carlofortino kazáda 'colostro' è anteriore al passaggio $-d->-\hat{r}$ - in sulcitano, documentato invece dalla variante calasettana kazára ${ }^{24}$. Anche ǵắle 'giogo per i buoi' e 'filare di vigna' mostra uno stadio antecedente alla caduta di $-l$-nel sulcitano antiochese, che ha attualmente ǵuái ${ }^{25}$.

5. Il contatto linguistico col sardo ha quindi inizio con la fondazione delle colonie, e fin da subito si presenta variegato e consistente; esso si appoggia all'introduzione di qualche elemento sardo nella fonetica e (forse) nella morfologia del tabarchino: ma l'introduzione nell' inventario fonologico tabarchino di una $\hat{r}$ intervocalica polivibrante apicale ${ }^{26}$, ad esempio, non ha creato sostanziali rivoluzioni nella struttura del dialetto, e, soprattutto, non ha creato interferenze nell'intercomprensione con i parlanti genovesi; anche l'introduzione relativamente frequente di voci con $-d$ - intervocalica non rappresenta una novità dirompente in un dialetto nel quale le desinenze in -ádu, -áda sono, storicamente, tuttaltro che rare ${ }^{27}$. 
Quanto alla neutralizzazione occorsa nel plurale dell'articolo indeterminativo femminile non è necessariamente imputabile a influsso sardo, come supposto, peraltro in forma dubitativa, da E. Blasco Ferrer ${ }^{28}$.

6. L'antichità e la quantità dei prestiti ridimensiona almeno in parte l'opinione, diffusa anche a livello popolare, di una maggiore ricettività verso i sardismi da parte della sottovarietà calasettana rispetto a quella carlofortina: questa opinione, appoggiata a considerazioni di ordine geografico ${ }^{29}$ appare smentita dalla presenza di un numero preponderante di sardismi comuni alle due località, e soprattutto dal rilievo dei sardismi specifici della parlata di Carloforte, in parte antichi in quanto assunti verosimilmente nella fase (1738-1769) successiva all'installazione della colonia tabarchina dell'isola di San Pietro e prima della fondazione di Calasetta ${ }^{30}$.

Che a Calasetta i prestiti legati alla terminologia rurale siano più numerosi e consistenti, sarà poi, in parte, conseguenza della tradizionale specializzazione economica della località, strutturata come si è visto sulla pratica intensiva della viticoltura.

Proprio a partire dalla specializzazione agricola di Calasetta è possibile ricostruire del resto, attraverso le fonti orali, alcune modalità di assunzione dei prestiti sardi.

La constatazione dell'esistenza di diverse voci legate ai lavori di campagna, tradizionalmente svolti da stagionali sardi ancora fin verso gli anni Cinquanta e Sessanta del sec. XX si spiega infatti (e viene spiegata dai locutori) col fatto che questi operai, dato il carattere precario della loro presenza in loco, non avvertivano l'esigenza di una maggiore integrazione linguistica nel contesto locale ${ }^{31}$ : erano i prupyetă $i$ tabarchini, in questo caso, a dotarsi di una conoscenza più o meno attiva del dialetto sulcitano o di quello della zona di Teulada dalla quale provenivano molti lavoratori stagionali ${ }^{32}$; era dunque naturale che la terminologia agricola risentisse di questa particolare situazione di diglossia, arricchendosi progressivamente di elementi lessicali sardi.

Attualmente quasi nessun tabarchino parla il sardo ${ }^{33}$, tranne, appunto, quegli anziani di Calasetta che lo appresero nei decenni passati per le esigenze di comunicazione con i braccianti impegnati nella raccolta dell'uva e in altre mansioni:

nyắtri $\mathrm{i}$ prími tệ́npi duvắimu parlắge $\mathrm{n}$ sắrdu, sệ́rte pắule ke nu sắimu se fắimu kapí... 1 ẹ̣a ćû́ fásile pe nyắtri parlắ w sắrdu ke lú w tabarkín... ẹmu pắule $\mathrm{k}$ ện difísili,

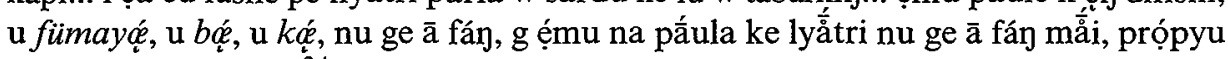
difisilísima: u šçóp $u^{34}$.

Il carattere «tecnico» dell'assunzione di molti prestiti trova quindi una spiegazione ancora in data recente, e appare significativo, in questo senso, il fatto che assai difficilmente le voci d'origine sarda si riferiscano ad aspetti generali delle pratiche agricole o dell'allevamento, limitandosi per lo più a concetti estremamente caratterizzati: i nomi della pecora e del gregge, ad esempio, rimangono genoves ${ }^{35}$, ma sono d'origine sarda denominazioni più specifiche, come quelle delle diverse fasce d'età del bestiame ${ }^{36}$. 
Un caso a sé è rappresentato in questo senso dal lessico vitivinicolo, che è quasi interamente sardo, probabilmente perché, a dispetto di un'opinione radicata, le tecniche di coltivazione e di produzione hanno sempre ripreso, come del resto è ovvio, modalità tipicamente sarde (di ascendenza catalana) piuttosto che continentali ${ }^{37}$.

Analogamente, la relativa abbondanza di battesimi dialettali di provenienza sarda (o catalano-sarda) per i pesci, aiuta a ridimensionare un altro luogo comune ricorrente, quello dei Tabarchini come popolo di consolidate tradizioni nel campo della pesca.

In realtà, malgrado la fortissima specializzazione marittima dei Carlofortini ${ }^{38}$, o fors' anche in ragione di essa, la pesca fu sempre considerata - ad eccezione della pratica industriale della tonnara - un'attività di sussistenza, oppure praticata per lo più da professionisti algheresi (il che potrebbe spiegare in parte l'abbondanza di sardo-catalanismi) e meridionali, Siciliani o Ponzesi ${ }^{39}$.

Un altro settore caratterizzato da una presenza significativa di sardismi, soprattutto a Carloforte, è infine quello del lessico espressivo, delle determinazioni di carattere, degli insulti e delle interiezioni, al quale sembra di poter associare la relativa frequenza di appellativi d'origine sarda nell' ambito del sistema soprannominale ${ }^{40}$ e l'utilizzo frequente di suffissi di provenienza campidanese, principalmente diminutivi e affettivi $^{41}$, ma anche peggiorativi ${ }^{42}$.

7. Nel tratteggiare un catalogo - certamente incompleto, ma nondimeno ampiamente rappresentativo - dei sardismi in tabarchino, è occorso tenere presenti alcune variabili significative, che arricchiscono la problematica della fenomenologia dei prestiti e introducono ulteriori spunti di riflessione: la corrispondenza semantica e fonetica tra voci liguri probabilmente già presenti nel fondo tabarchino originario e analoghe forme campidanesi; l'esistenza di ligurismi (non di rado «tabarchinismi») in campidanese e in particolare nella parlata sulcitana di Sant'Antioco; il ruolo recente dell'italiano regionale come eventuale veicolo di alcuni elementi lessicali (e non solo) sardi in tabarchino.

Nel primo caso, le pur minime concordanze tra genovese e campidanese rischiano di creare equivoci tali da incrementare indebitamente il repertorio dei sardismi in tabarchino ${ }^{43}$; concordanze lessicali del tutto casuali, soprattutto se si verificano in campi semantici nei quali l'apporto sardo sia particolarmente ricco, assumono un'importanza diversa, perché in tali casi il gioco delle sovrapposizioni e delle interferenze si rivela più complesso del mero trapianto lessicale o del mero calco semantico ${ }^{44}$.

Di particolare rilievo sono inoltre le concordanze nell'assunzione per via indipendente di elementi lessicali di provenienza ispanica (catalana o castigliana) ${ }^{45}$ o france$\mathrm{se}^{46}$, che richiedono un'attenta considerazione della cronologia e una riflessione accurata della fenomenologia generale dei sardismi in tabarchino, prima che si possa stabilire con sufficiente approssimazione se la voce in questione è provenuta dalla Liguria o se è stata assunta più recentemente in virtù dei contatti col retroterra.

Per quanto riguarda i ligurismi in sardo, argomento toccato solo marginalmente dal Wagner con particolare riferimento al dialetto antiochese ${ }^{47}$, essi creano assonanze che 
possono aumentare l'impressione di un apporto diversificato e consistente dal sardo al tabarchino, ma che testimoniano piuttosto l'importanza economica e la rilevanza sociale assunta dai coloni liguri nel contesto sulcitano ${ }^{48}$.

L'apporto dell'italiano regionale sardo, infine, codice pienamente condiviso dai locutori tabarchini, può avere contribuito alla diffusione recente di modismi e di locuzioni gergali che dovrebbero essere tenute su un piano diverso rispetto ai sardismi veri e propri ${ }^{49}$.

\section{Glossario}

L'elenco che segue si basa sui materiali inediti da me raccolti presso le comunità tabarchine di Carloforte e Calasetta per il progettato Dizionario etimologico-storico tabarchino (DEST) ${ }^{50}$.

La presentazione che viene offerta è per forza di cose selettiva, ma può essere considerata ampiamente rappresentativa, come si anticipava, del corpus delle voci di origine sarda presenti nel lessico tabarchino: come criterio generale, per la campionatura presentata in questa sede sono state scelte quasi esclusivamente voci che trovassero corrispondenza nei vocaboli inventariati in alcune opere fondamentali della lessicografia e della linguistica sarda, il vecchio Vocabulariu dello Spano ${ }^{51}$, La vita rustica in Sardegna ${ }^{52}$ del Wagner, la già citata opera dello stesso autore, La lingua sarda (= Ls) e, naturalmente, il suo Dizionario Etimologico Sardo $(=\mathrm{DES})^{53}$ : delle voci ci si limita a segnalare l'origine sarda senza ulteriori approfondimenti etimologici che non risultino significativi per il commento della voce in questione e per la sua contestualizzazione nel lessico tabarchino.

I sardismi così individuati ${ }^{54}$ sono stati classificati in categorie semantiche più o meno ampie e corredati, quando necessario, di brevi commenti. La sigla TB indica che la voce è comune al lessico delle due comunità, $\mathrm{CA}$ che è stata raccolta a Calasetta, CF a Carloforte ${ }^{55}$. Il riferimento all'ALI segnala che la voce in questione è presente nei materiali dell'Atlante Linguistico Italiano ${ }^{56}$ ma che risulta completamente uscita dall'uso vivo oppure (e in questo caso ciò viene esplicitamente segnalato) che essa viene soltanto ricordata dagli informatori. La grafia delle voci tabarchine è quella, come anticipato, del LEI; per le corrispondenti voci sarde si è mantenuta la grafia delle fonti, con alcune semplificazioni, dovute a motivi tipografici, per il sistema fonetico del Wagner ${ }^{57}$. Le sigle utilizzate sono di facile scioglimento.

\section{a) Suolo e ambiente. Animali selvatici di terra}

Nel campo del lessico relativo all' ambiente le voci d'origine sarda sono forse meno frequenti di quanto ci si potrebbe attendere in un ambito semantico strettamente connesso ai luoghi, e quindi, almeno in teoria, suscettibile di arricchirsi di voci indigene al momento della colonizzazione. Va però ricordato che la sede originaria dei Tabarchini in Tunisia non presentava forme del suolo e un tipo di vegetazione e di fauna troppo diversi da quelli incontrati in Sardegna, e che pertanto il lessico ligure potè conservarsi in gran parte. È ancora quella originaria, ad esempio, gran parte della nomenclatu- 
ra relativa alle essenze della macchia mediterranea, e anche i nomi di uccelli e altri animali, salvo adeguamenti semantici ${ }^{58}$, sono prevalentemente liguri. Appartengono all'adstrato sardo, invece, alcune voci legate all'organizzazione del suolo.

1. benásu, s.m. CA (ALI) 'brughiera' - SpanoI,257 benàzzu 'acquitrino, palude'. Forse l'informatore dell' ALI equivocò sul significato del quesito propostogli.

2. ćurín, s.m. CA 'mignattino dalle ali bianche' - Pare una variante di zurruliu (SpanoII,426) 'nome di diversi uccelli', DES (II,602) tsurrulíu ${ }^{59}$, forse con influsso di čúrru 'zampillo', DES $(I, 455)$ : il mignattino è una sorta di gabbiano che si tuffa in mare. Per la fonetica sulcitana, come noto, $t s$ del campidanese comune $>\dot{c}$.

3. fệwra, s.f. CF 'ferula, pianta erbacea' - Spanoll,26 feurra, DES (I,514) féurra s.v. férula.

4. fî́ge murî́ke, s.f. pl. CF 'fichi d'india'; meno usato rispetto al tipo lig. fíge díndya. A CA prevalentemente muštáći - Vr74, DES (I,520) figu muríska.

5. furyadrộ́žu, s.m. TB 'fabbricato rurale': CF 'abitazione con podere', CA piuttosto 'magazzino'; voce di scarso uso, e riferita comunque al retroterra sardo - SpanoII,48 furriadroxu 'ritiro, tenuta', DES (I,561) furriadróžu 'capanne di pastori ed ovile, di cui molti divennero col tempo piccole popolazioni e comuni'.

6. gurệu, s.m. TB 'cardo selvatico' - SpanoII,69 gurèu, DES (I,300) guréu.

7. inperdáu, s.m. CA 'selciato'; voce poco usata rispetto al sinon. risứ di tipo lig., comune anche a CF - SpanolI,85 imperdai 'selciare'.

8. kugû́ra, s.f. CF 'gorgoglione, forfecchia'; voce di scarso uso - SpanoI, 368 cugùrra 'forfecchia'; DES $(I, 419)$ 'bruco, forfecchia'.

9. kukumệ́u, kumệ́ $u$, s.m. CA 'un uccello notturno' o forse 'denominazione generale degli uccelli notturni' (ma la 'civetta' è suyéta come a CF) - SpanoI,366 cuccumeu 'civetta', DES $(1,416)$ kukkuméu s.v. kukkumiau 60 .

10. kusộ́rža, s.f. CF 'distretto rurale'; non comune e riferito per lo più all'ambiente sardo; a CA Cussòrgia è una frazione abitata da un centinaio di persone di origine sarda - Spanoll,376; Vr68,259, DES (1,439) kussòrdza.

11. lû́a, s.f. TB 'varietà di euforbia' - SpanoII,196 lua, DES (I,40) lúa.

12. munǵédu, s.m. CF 'chiocciolina'; a CA il termine è conosciuto come voce sarda - SpanoII,231 mungiètta 'chiocciolina', DES (II,126) mondzètta, monğètta (s.v. móndzu).

13. nuắržu, s.m. CF 'nuraghe'; voce uscita dall'uso - dalla variante campidanese del tipo nuráke: nuráži, DES (II,176), con metatesi di -r-.

14. nurẳgu, s.m. CA 'nuraghe' $\mathrm{e}$ 'tipo di vitigno'; $\mathrm{CF}$ 'solo 'vitigno' - SpanoII,244 nuràgus 'uva trebbiana', DES (II,179) nurágus. Per il sign. di 'nuraghe' deve essersi verificata una confusione tra il nome del vitigno e quello del manufatto architettonico.

15. nužẹeda, s.f. CA 'nocciòlo' e 'nocciola'; il sinon. di tipo lig. nisếa è conosciuto solo come voce del dialetto di CF, ove è d'uso corrente - Ls104-105, DES $(\mathrm{II}, 174)$ nužèdḍa.

16. puvúpu, s.m. CA 'upupa'; forma d'uso corrente rispetto al tipo lig. galétu de mấrsu, comune invece a CF, che a CA è usato prevalentemente in senso scherzoso ( $u$ fa u galẹ́tu de mấrsu, 'vuol mettersi in mostra') - SpanoII,301 pubusa 'upupa', DES (II,327) pubúsa, ma puvúppa è forma sulcitana (nostre informazioni). 
17. sẹ́sinu, sẹ́sine, s.m. CA, sẹ́sanu CF, 'giunco di padule' CA; 'loglio' CF - SpanoII,353 sèssini 'cipero, caretto (erba)'; Ls263, DES (II,412) su șèssini 'giunco marino'.

18. škanpyấu, agg. CF solo nella locuz. terên škanpyấu 'radura' - SpanoII,340 scampiòsu 'spazioso' .

19. tấnka, s.f. TB 'podere chiuso'; voce di uso corrente - Spanoll,384 tànca 'podere' Vr68 tánka, DES (II,463) s.v. tankare.

20. tapắda, s.f. TB 'tipo di chiocciola' - SpanoII,384 tapada, DES (II,465) tappáda 'lumaca che sta sottoterra, chiocciola'.

\section{b) Agricoltura in generale. Aratro. Coltivazione dei cereali}

Quello agricolo è, come è stato anticipato, il settore del lessico in cui si riscontra con maggiore frequenza l'apporto sardo nel tabarchino. Le tecniche agrarie di tipo estensivo furono certamente apprese dai coloni dopo il loro trasferimento in Sardegna: nella Liguria costiera (e tanto meno a Tabarca, evidentemente) non veniva praticato l'uso dell'aratro, e di conseguenza tutto il lessico relativo è stato mutuato dal sardo, così come la terminologia relativa alla coltura cerealicola, che non rientra minimamente nelle tradizioni agricole liguri. Significative anche alcune assenze, come il tipo prato, comune al lig. e al sardo, sostituito completamente da campo.

21. arǵólla, s.f. TB 'aia' - SpanoI,210 arzòla, Vr115, DES $(\mathrm{I}, 112)$ arğòla.

22. arǵoláda, s.f. TB 'aiata' - Vr119 ardzoláda.

23. asurká, v. intr. TB 'far solchi' - Spanol,216 assurcài; resta impossibile stabilire se súrku debba essere considerato o meno un prestito sardo, ma la forma verbale è assente dai lessici lig.

24. deytắle, s.m. CA 'ceppo dell' aratro in legno', 'vomere' - Spanol,382 dentàli; Vr92, DES $(\mathrm{I}, 461)$ sa dentále; il cambiamento di genere sarà dovuto all'influsso dei numerosi nomi in - le di derivazione dotta.

25. fẹ́rtu, agg. TB 'non sviluppato, stentato, detto di pianta' e in senso fig. anche 'di persona' - SpanoII,25 fèrtu 'ferito, còlto, vulnerato, scagliato, percosso', DES $(\mathbf{I}, 513)$ part. pass. di fèrrere, ferriri 'ferire, colpire'

26. kaminệa, s.m. TB 'estremo lembo non coltivato del campo' e, per estensione, 'sentiero' - Spanol,299 caminèra 'androne, andito, viale', DES (1,275) kaminèra, s.v. kam(m)ínu. L'adeguamento fonetico, con $r>\emptyset$ potrebbe denunciare l'antichità del prestito.

27. kána, s.f. CA 'stelo del granturco' - Spanol,303 canna de trigu, d’orzu 'stelo', DES (I,283) kánna de gáuli, de láttia; il tipo canna per 'stelo' non è noto in area lig.

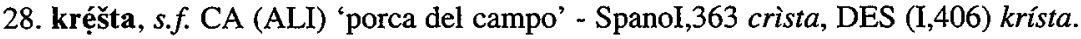

29. mandefếru, s.m. CA 'rastrello' - a Sant' Antioco ũna mấu de verru 'rastrello con quattro o cinque denti', DES (II,69), s.v. mánu.

30. mánega, s.f. TB 'covone' - SpanoII,207 màniga de trigu 'covone'; Vr112 mániga, cfr. anche DES (II,66) s.v. mánika. Calco semantico.

31. manúngulu, s.m. CA 'mannello di frumento' - Vr112, DES (II,70) man(n)úgu. La mancata riduzione del proparossitono potrebbe denunciare l'antichità del prestito.

32. manugká́, v. tr. CF 'scalzare' - SpanoI,147 ammanuncài 'malmenare, palpeggiare, brancicare, gualcire, toccar colle mani, comprimere'. Specializzazione semantica a partire dal concetto di 'alterare, rimuovere'. 
33. mẳra, s.f. TB 'zappa piana' - SpanoII,211 marra, DES (II,77) 'zappa, marra'. Il tipo mấra è noto anche in Liguria, ma non è usuale.

34. marajćúy, s.m. TB 'zappone' - accrescitivo di marra 'zappa': cfr. marráćću, DES (II,77), s.v. marra.

35. mulọ́ni, s.m. CA 'termine di confine tra i campi'; concorre la forma lig. tếrmine - Spanoll,231 mullòne 'limite, confine'; Vr87, DES $(\mathrm{II}, 136)$ mullòni. Esempio tipico di sardismo non assimilato morfologicamente (< cat. molló).

36. muštayộni, s.m. CA 'spaventapasseri'; in passato la voce era usata anche per indicare il 'fantocio di carnevale' - SpanoII,235 mustajòni 'spauracchio, fantoccio'; Vr110, DES (II,146) mustayòni.

37. nẹrvẹ́du, s.m. CA 'coltello dell' aratro di legno'; da informazioni indirette risulta che una ventina d'anni fa si conosceva anche la variante nẹrvẹ́lu, con adeguamento alla suffissazione di tipo lig. - Vr92 nérbyu, cfr. anche DES (II,162) s.v. nérvinu.

38. paštệ́a, s.f. TB 'aiola', soprattutto di tipo decorativo, ma anche 'semenzaio' - SpanoII,264, DES (II,232) pastèra 'aiuola, cassettone di fiori'. Con adeguamento al tipo lig. $r>\phi$.

39. pizẹ́di, s.f. CA ‘cicerchia' - SpanoII,282 pisèddu, DES (II,283) pișédḍ, s.v. píșu.

40. purtážu, s.m. CA 'bica di fieno o frumento' - Voce assente nei repertori consultati.

41. škúba, s.f. CA 'scopa grossolana usata per pulire l'aia' - SpanoII,140 iscòba; Vr116 iskòba 'scopa per pulire l'aia'. La forma skúba è presente in lig. per 'scopone, gioco di carte'.

42. štệve, s.f. TB 'stegola dell' aratro' - Vr92,94 stèva.

43. tepperadrộ́žu, s.m. CA (ALI) 'coltello dell'aratro di legno'; il termine si ricorda, ma non viene più usato - SpanoII,386 temperadròxu de orbada 'cappio del vomere', DES (II,473) temperadróžu s.v. temperare.

44. trabúsu, s.m. TB 'forcone' - SpanoII,399 trebuzzu; Vr119 trebúttsu; DES (II,521) s.v. trivúćcu cita le varianti campid. treúttsu, trebússu e, a Sant'Antioco, trafúčču.

45. vakánte, agg. CA (ALI) 'di terreno lasciato in riposo ma che viene rivoltato' - Vr70 bacantes (nei documenti antichi; mod. piuttosto vagantíu. La forma dovette appoggiarsi all'agg. vakánte 'vuoto, svuotato in senso generico', che dạ più indizi sembra però un prestito del siciliano penetrato in TB attraverso il lessico della tonnara.

\section{c) Viticoltura e vinificazione}

La viticoltura rappresentò la principale fonte di benessere per Calasetta fino all'inizio della seconda metà del sec. XX. Il vino prodotto veniva trasferito a Genova su imbarcazioni provenienti dalla riviera ligure (i cosiddetti rivắni) ed esportato in Italia e in Francia, dove veniva usato per «tagliare» prodotti più nobili. Le tecniche di coltivazione della vite e di produzione del vino, che riflettono le modalità catalane diffuse in Sardegna, furono apprese certamente in loco dai coloni, e l'utilizzo massiccio di manodopera sulcitana e campidanese ebbe come conseguenza, secondo le modalità già chiarite, il radicamento di un lessico settoriale di prevalente origine sarda. Restano tuttavia liguri, come in altri campi semantici, le denominazioni generali della pianta, dei processi di produzione, della maggior parte degli strumenti e dei recipienti impiegati per la vinificazione. 
46. amēlá, v. tr. CA 'scolmare un tino eccessivamente pieno mediante una cannuccia' - SpanoI,149, DES (I,81) ammellare 'cessare, placare' (voce logud.). Specializzazione semantica di un termine d'uso generale.

47. barbatá́, v. intr. CA ‘dissodare' - Vr78,83, DES (I,177) brabattai s. v. barbáttu, logud. (b)arbattare.

48. brásu, s.m. TB 'il tralcio che viene lasciato dopo la potatura affinché germogli' - Vr113,197 ráttu de íde (= 'braccio di vite') 'tralcio'; cfr. anche DES (I,224) s.v. brá $\theta \theta u$. Calco semantico: il tipo è assente in area lig.

49. buvấle, s.m. TB 'varietà di uva nera - Vr204, DES (I,221) bovàle 'specie di uva nera' (cat. boval).

50. ćása, s.f. TB 'interfilare' - Vr196 práttsa 'spazio libero tra due filari', cfr. anche DES (II,304). Non risulta in area lig. questa specializzazione di PLATEA.

51. filevệru, s. $m$. TB 'acquavite' - Viene considerata denominazione recente a partire dalla nota denominazione sarda: in passato la grappa non era prodotta, né risulta che se ne facesse particolarmente uso.

52. ǵirọ́, s.m. TB 'nome di un vitigno' - Vr206, DES (1,607) ğirò 'diversi tipi di uva'61.

53. ǵwăle, s.m. TB 'filare di vigna' - SpanoI,60 giuàle; Vr196, DES (I,711) ğuáli de (b)ínğa, s.v. yugu.

54. karegadrộža, s.m. TB 'tralcio occhiuto, sopraccarico', ma CA anche 'qualsiasi tipo di carico eccessivo' - Vr198 karryadròz̆a 'tralcio che nella potatura viene lasciato intatto', DES (I,307) karriadròža 'tralcio pieno di gemme'.

55. kubedína, s.f. TB 'tino, botte'; voce d'uso - SpanoI,366 cubedina; Vr139 kubèdda 'botticella'; Vr202 kubidina 'tino di legno', DES $(\mathrm{I}, 434)$ kubedina s. v. kúpa.

56. maruná, v. tr. TB, amaruná CA, v. intr. 'dissodare, sarchiare', detto in genere della vigna; SpanoII,211 marronài 'zapponare'; Vr79, DES (II,77) marrai 'zappare', s.v. márra; Vr88, 189 marrone 'zappa'; cfr. la voce má̀ra al punto b).

57. mọ́nika, s.f. TB 'varietà di uva rossa' - SpanoII,226 mònica 'uva canaiola', DES (II,125) mòni$k a$. I vitigni coltivati a CA sono gli stessi che si piantano nel Campidano.

58. pilwín, s.m. pl. CA (ALI) 'la parte della pianta rimasta nella terra, se mette nuovi polloni' SpanoII,279 pilloni 'germoglio, rampollo, virgulto', DES (II,331) pillòni s.v. pudzòne. La forma registrata per l'ALI presuppone un sing. *pilún, e di conseguenza un tipo di adeguamento morfologico piuttosto insolito nel passaggio dal sardo al TB.

59. pudọ́ni, s.m. CA 'getto infruttifero della vite' - SpanoII,302 pudòni 'occhio a frutto del sermento'; Vr197.

60. sekreštá, $v$. intr. TB 'capitozzare, effettuare la prima potatura' - SpanoII,350 segrestare 'sfemminellare, tagliar le viti inutili'; Vr198, DES (II,400) segrestai (<cat. segrestar), s. v. segrestare.

61. sermệntu, s.m. TB 'sarmento' - SpanoII,353 sermèntu; cfr. Vr196, DES (II,384). Tipo assente in Liguria.

62. širá, v. tr. TB 'accorciare i tralci, diramare, spampanare' - Spanoll,345 scirrài; Vr198, DES (II,343) širrai s. v. kírra.

63. škarsá, v. intr. TB 'scalzare la vigna' - SpanoII,341 scarzài; Vr198 skartsai. 
64. žmamá, v. intr. TB 'tagliare parte dei tralci per dare forza alla vite' - SpanolI,154 ismamàre; Vr198 șmammai.

d) Allevamento degli ovini, dei caprini e dei suini

L'accorpamento in un unico paragrafo delle voci legate al bestiame di taglia media intende anche sottolineare la scarsa importanza economica, presso le comunità tabarchine, dell'allevamento delle pecore. Ancor oggi le greggi presenti a CA sono per lo più di proprietà di pastori sardi, o possedute a sósyu (in società) fra agricoltori tabarchini e pecorai provenienti dal Sulcis. Di conseguenza, la terminologia d'origine sarda è preponderante, malgrado siano genovesi i termini d'uso più generale. La presenza di alcune voci di area prevalentemente logudorese potrebbe essere legata, qui come nella sezione e), alla dinamica degli spostamenti interni delle greggi e dei pastori in Sardegna.

65. akurá, $v$. tr. TB 'radunare un gregge in luogo chiuso' e per estensione 'rinchiudere, precludere la fuga' - SpanoI,98 accorrài 'radunare, unire il bestiame', DES (I,387) akkorrare, akkorrai, s.v. kórru

66. amindá, v. intr. CA 'far pascolare il gregge su terreni adiacenti ai seminativi, durante il lavoro agricolo' - Spanol,149 ammendàre, ammindài 'foraggiare, pascere il bestiame dòmito'; DES (I,103) ammindai (s.v. mendare) 'pascere il bestiame domito nella minda o chiuso riservato, vacuo nei seminativi', a Sant'Antioco.

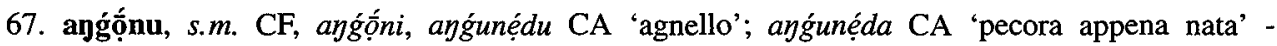
SpanoI,160 'agnello'; Vr242 anğòni, 244 anğonéḍ̣ mento morfologico parziale.

68. ćirọ́tu, s.m. CA 'stalletta per i capretti' - SpanoI,332 cirra 'mandra degli agnelli e dei capretti'; Vr254, DES $(\mathrm{I}, 343)$ čirra 'recinto coperto per mettervi i capretti', s.v. kírra.

69. coó cọ́, escl. CA 'richiamo per avvicinare il maiale' - Spanol,331 ciò 'voce con la quale si chiama il porco'; Vr256 čo čo, DES $(\mathbf{I}, 453)$ čo.

70. fuí, v. intr. TB 'fuggire, essere irrequieti, detto del bestiame in amore' - SpanoII,45 fuire 'fuggire', DES (1,554) fuíri, s.v. fúgere.

71. inkrabištá, v. tr. CF 'impastoiare' - SpanoII,99 incrabistài 'mettere il capestro'; cfr. DES (I,399) s.v. krapistu.

72. karaláti, s.m. CA 'malattia virale che fa calare il latte agli ovini' - da karái, variante sulcitana di kalái, DES (I,266) 'calare' e látti 'latte'.

73. kộrrdula, s.f. CF 'treccia di budella di pecora'; la si considera una vivanda di tradizione sarda SpanoI,355 còrdula; Ls139, DES (I,380) kórdula 'intestini della capra o della pecora arrostiti sulla cenere e intrecciati'.

74. krabîštu, s.m. TB 'capestro' - Spanol,360 crabistu; Vr226 krabístu, DES (1,399) krapístu.

75. kulúdu, agg. TB 'di animale (per lo più il verro) non castrato, atto alla riproduzione' - SpanoI,339 colludu 'intiero, non castrato'; Vr225,256, DES (I,395) kollúdu (pórku gollúdu), s.v. kòdza.

76. kurî́a, s.f. CA 'collare del campano' - Spanol,356 corria, DES $(1,385)$ korría 'correggia'; specializzazione semantica della voce nel passaggio al TB.

77. kúrte, s.m. CA 'spazio aperto dove pernottano le pecore' - Spanol,358 corte de arveghes 'pecorile'; Vr248 kòrti 'ovile', cfr. DES $(1,389)$ s.v. kòrte; la voce significa anche 'cortile'. 
78. lyunẹeda, s.f. CA 'zufolo di canne dei pastori' - Vr341, DES (II,16) liunèḍ̂as 'launeddas'; voce pressoché uscita dall'uso: per il tipico strumento sardo, che localmente si conosce solo dopo la recente affermazione nell'ambito del folk revival, si usa la forma launéddas dell'italiano regionale.

79. meǻ, intr. CA 'belare' - Spanoll,216 melare, DES (I,192) melare, melai s.v. belare: 1'adattamento fonetico fu forse facilitato da un'antica presenza del tipo lig. beá, sul quale la voce sarda si sovrappose.

80. medắu, s.m. TB 'ovile aperto'; voce conosciuta, ma utilizzata quasi esclusivamente con riferimento all'organizzazione pastorale del Sulcis - SpanoII,201 madàu 'ovile'; Vr249, Ls130, DES (II, 98) medáu.

81. sakắya, s.f. TB 'agnella di un anno' - SpanoII,333 saccàju; Vr244, Ls256, DES (II,374) sakkáyu 'agnello di un anno'.

82. sementúza, s.f. CA 'pecora di un anno che non ha figliato' - SpanoII,351 sementòsu 'agnello di due anni'; Vr244 sementúșu 'pecora fino a due anni', DES (II,402) sementóṣu s.v. semertóșu.

\section{e) Produzione casearia}

Fu sempre irrilevante nel sistema produttivo tabarchino, e legata all'uso domestico e locale; dal porto di Carloforte e dalla marina di Calasetta partivano periodicamente per Genova e per altre destinazioni del continente carichi di furmáǵu sắrdu, la cui denominazione chiarisce già, di per sé, tuttavia, la provenienza dal retroterra.

83. aî́šku, s.m. CA 'forma per il formaggio, fiscella' - Spanol,127 logud. aiscu 'scodella, scodellino per mettere il formaggio fresco, cascino'; Vr268, DES (I,470) aísku logud. (s.v. dísku) 'forma per il formaggio', campid. dískua.

84. furmáǵu mắrsu, s.m. CA 'tipo di formaggio molle piccante' - Vr273 káșu márču 'formaggio marcio'; calco semantico.

85. gọ́du, s.m. CF 'ricotta fermentata' - SpanolI,58 giòddu 'latte coagulato cui si dà l'acido'; Vr273 ğóddu 'ricotta', DES $(\mathrm{H}, 709)$ yóḍ̣ $u$ 'una specie di latte fermentato acido che si condensa in un coagulo molle e omogeneo'. Per 'ricotta' in senso generale si usa la forma lig. rekớtu 62 .

86. kalá, akalá, v. intr. CA 'cagliare'; forma d'uso corrente rispetto al più raro aprếydise, tipo lig. diffuso a CF - SpanoI,297 calliài, DES (I,397) kallai s.v. kragare.

87. kálu, s.m. CA, kálaw CF 'caglio' - SpanoI,297 callu; Vr267,274, DES (I,397) kállu. L'ampliamento della desinenza nella variante di $\mathrm{CF}$, di carattere analogico, è caratteristico in genere dei nomi di piante o animali: fǘnzaw 'fungo', kúnbaw 'colombo', ecc.

88. kazáda, s.f. CF, kazára CA 'colostro' e 'il formaggio che se ne ricava' - SpanoI,313 casàda 'formaggio cotto'; DES $(\mathrm{I}, 317)$ kașáda 'formaggio cotto' s.v. káṣu il diverso trattamento di - $d$ rivela l'antichità del prestito nella varietà di $\mathrm{CF}$.

89. kắžu, s.m. CA 'formaggio fresco' - Spanol,316 casu 'formaggio in genere'.

90. murígu, s.m. TB 'latte cagliato pronto per fare il formaggio' a CA; CF, CA 'tramestio' - Cfr. murigá al punto t): murigai 'rimenare il formaggio' in DES $(\mathrm{II}, 139)$ s.v. murikare. 


\section{f) Allevamento dei bovini}

Ebbe soprattutto in passato una discreta importanza a Calasetta, dove alla fine del sec. XIX si costituì persino, tra i proprietari di buoi da trasporto, la cosiddetta kuppañía di bớ, una delle prime società di mutuo soccorso attive in Sardegna.

91. bai, escl. CA 'vai!, incitamento per i bovini' - Voce del verbo andái, usata solo in questa accezione.

92. berẳnu, s.m. CA, barắnu $\mathrm{CF}$ 'la stagione agricola', ma intendendo specialmente 'la stagione durante la quali i bovini crescono, sono all'ingrasso, pígay $u$ b.' - SpanoI,260 berànu, DES (II,571) veránu 'primavera'. Specializzazione semantica. Per 'primavera' si usa normalmente la forma lig. primaváya.

93. bọ́nu, escl. CA 'calma!, esortazione per i buoi' - voce d'uso, penetrata talvolta anche nella conversazione corrente: 'sta' calmo', detto a persona.

94. bwenắržu, s.m. TB 'bifolco, bovaro' - SpanoI,272 boinàrgiu 'boaro', DES $(\mathrm{I}, 216)$ boinárğu s.v. boínu; usato spesso come moderato insulto per 'persona maleducata, incivile'.

95. fuštî́gu, s.m. TB 'spranga del carro a buoi' - SpanoI,49 fustigu, DES (I,563) fustígu 'fuscello'; rifletterà forse una denominazione locale dell'area sulcitana.

96. grüminá, v. intr. TB 'ruminare' - SpanoII,67 grumiài, DES (I,368) aggrumiai s.v. rumigare 'ruminare'; sembra essersi verificata la sovrapposizione della voce sarda sul tipo ruminare, non estraneo all' area lig.

97. ǵúnǵe, v. tr. CA 'aggiogare' - SpanoII,61 giùngiri; Vr102, DES (1,713) ğúnğiri iṣ bòis; la voce lig. (a)zúnze è d'uso corrente nel senso generale di 'unire', 'congiungere'.

98. ǵwắle, s.m. TB 'giogo per i buoi' - SpanoII,60 giuàle; Vr100,140, DES (1,711) ğuáli; a Sant'Antioco ğuái 63 .

99. láku, s.m. TB 'truogolo in pietra per i bovini, abbeveratoio' - SpanoII,181 làcu; Vr139, DES (II,4) lák(k)u; il tipo lig. trạ́ǵu vale 'abbeveratoio' in generale.

100. lamún, s.m. CA 'secchio per mungere' - Vr217, DES (II,6) lama; malgrado la probabile origine continentale della voce sarda, questo tipo è sconosciuto in Liguria in questo specifico significato.

101. lọ́la, s.m. TB 'stalla'; anche 'loggia per riporvi il carro' - SpanoII,195 lòlla 'loggiato, tettoia', DES (II,39) s.v. lòdza; con specializzazioni diverse ricorrono anche i tipi lig. štála e štáǵu.

102. lộru, s.m. TB 'giuntoia di cuoio per legare il bue al giogo' - SpanoII,196 loru 'correggiolo'; Vr101, Ls129, DES (II,38) lòrus 'corregge per fissare il giogo'.

103. malọ́ru, s.m. TB 'giovenco, torello'; anche malọ́ra 'giovenca' - Spanoll,205; Vr212 mallóru 'vitello di circa un anno', cfr. DES (II,55) s.v. mağğólu.

104. malurẹ́tu, s.m. CA 'vitello di 9 o 10 mesi' - SpanoII,205 mallòru 'toro, giovenco'; si noti l'inusuale adeguamento al suffisso dimin. di tipo lig.

105. manáda, s.m. TB 'budello bovino' - Vr112 manáda.

106. masáme, s.m. CA 'interiora del bue' - SpanoII,215 mazzàmini 'interame, interiora'.

107. mažídu, agg. CA 'mansueto, pacifico', detto di bovini e in senso fig. di persone - SpanoII,212 masèdu; Vr226 mașédu 'mansueto', DES (II,85) mașétu. 
108. muzúnǵu, s.m. TB 'cibo addizionale che si dà ai buoi per incitarli' e fig. allettamento'; CF anche 'bolo di cibo, masticamento continuo' - Spanoll,236 musùngiu 'razione dei buoi'.

109. ordinắgu, s.m. $\mathrm{CF}$, ordenágu $\mathrm{CA}, s . m$. 'redini dei buoi' (pl. ordenágíi) - SpanoII,249 ordinàgus 'redini'; Vr102 ordinágus, cfr. DES (II,343) s.v. redrinákos.

110. štrúmbulu, s.m. TB 'pungolo' - SpanolI,372 strùmbulu, Vr104, DES (II,436) strúmbulu, a Sant'Antioco strumbu (nostre informazioni).

111. títe, s.m. CA 'petto della mucca' - SpanoII,392, DES (II,489) titta 'mammella'; termine di discreto uso, adoperato talvolta anche per 'seno muliebre' in concorrenza con la forma lig. tetín.

\section{g) Il carro}

All'epoca d'oro della viticoltura calasettana, i carri per il trasporto dell'uva durante la vendemmia venivano noleggiati nel retroterra sardo e soprattutto nella zona di Teulada. Ciò provocava il trasferimento di decine e decine di lavoratori avventizi (ironicamente detti pariǵín 'parigini in vacanza'), che il più delle volte pernottavano sui loro veicoli parcheggiati lungo le strade della cittadina 64 . 112. ayộni, s.m. TB 'specie di cuscinetto infilato nel timone del carro e al quale si adatta il giogo'; voce uscita dall'uso - Spanol,127 ajòne 'gombìna, ordigno di pelle che si attacca al timone dell'aratro'.

113. áša, s.m. CA 'sala, asse del carro' - Vr179,181, DES (I,142) ašya.

114. bútu, s.m. CA 'mozzo della ruota' - Spanol,288 bùttu; Vr182, Ls248, DES (I,249) búttu; in Vr, il Wagner proponeva una derivazione da cat. botó, in Ls e DES preferisce piem. but. Ad ogni modo, la voce è assente in Liguria.

115. mekánika, s.m. CA 'martinicca' - Vr184, DES (II,98) logud. mekkánika, camp. matánika.

116. šệrdya, s.f. TB 'stuoia, graticcio che fa da sponda al carro a buoi' - Spanol,321 cèrda; Vr186, Ls112-113, DES $(1,446)$ čèrda.

117. tráka, s.m. CA 'carro a buoi che si prendeva in affitto per i pellegrinaggi a Sant'Antioco e a Tratalias'; voce uscita dall'uso - Spanolf,395 tracca 'carro a baracca', DES (II,502) trákkas 'carri coperti con tende'.

\section{h) Allevamento degli equini. Animali da soma e loro corredo}

Il possesso di un asino (più raramente di un cavallo) era frequentissimo in passato: occorre pensare che i coltivi e le vigne erano spesso a grande distanza dal centro abitato, sia a Carloforte, ove l'agricoltura era un' attività complementare (ma pur sempre importante), sia a Calasetta, ove essa rappresentava l'attività principale della stragrande maggioranza della popolazione. Disporre di un mezzo di trasporto era quindi una condizione essenziale per il lavoro nei campi.

118. arbúga, s.f. CA (ALI) 'barbazzale' - SpanoI,186 arbùle logud. 'barbazzale', DES (I,177) s.v. bárba cita le forme arbúle e arbúda.

119. bệrtula, s.m. TB 'bisaccia, sporta, gerla' particolarmente quella dell' asino; CA anche 'vesciche, sacche di pus sotto la pelle' - SpanoI,261 bèrtula 'bisaccia'; Vr232, DES (I,198) bértula.

120. beštyọ́lu, s.m. CA 'nome affettivo e familiare dell'asino' - SpanoI,262; Vr140 bestyólu 'asino', cfr. DES $(I, 199)$ s.v. béstia ${ }^{65}$.

121. ẹ́gwa, s.f. CA 'cavalla' - SpanoI,410 ègua; Vr222, DES $(\mathrm{I}, 485)$ ègwa 'cavalla'; a CF kavála. 
122. mará, s.f. CA 'calcio dato dal cavallo con le zampe anteriori'; voce pressoché uscita dall'uso SpanoII,211 marràda 'colpo di zampa'; Vr234 peșaișì a mmarrádas; Vr237 márra 'unghia, zoccolo'; DES (II,77) marrai 'scalpitare'.

123. retránga, s.f. TB 'finimento sottocoda dell' asino o del cavallo' - SpanoII,324 retrànga 'posoliera'; Vr227 (ar)retránga, cfr. DES (II,357) s.v. rètránka.

124. štríǵula, s.f. CA 'striglia' - SpanoII,371 strìgiula; Ls190 striğğulai, DES (II,699) stríğğula.

\section{i) Apicoltura}

Le comunità tabarchine non vantano tradizioni nel campo dell'apicoltura, che in passato fu praticata soltanto occasionalmente. Il lessico relativo è quasi tutto d'origine sarda, a partire dal nome stesso dell' 'ape' a Calasetta. Per un caso incerto, cfr. nota 41.

125. ábis CA (ALI), ábisi, ábizi, s.m. 'ape' - SpanoI,85 abi; Vr208, DES (I,98) ábi s.v. ápe ${ }^{66}$.

126. abyọ́ni, s.m. CA 'calabrone' - Tipo non documentato sui repertori sardi consultati.

127. kažídu, s.m. TB 'alveare'; CF fig. anche 'persona con la testa tra le nuvole, che ha qualcosa che gli ronza nel cervello' - Spanol,315 casiddu; Vr208, DES (I,312) kașídḍ ${ }^{67}$.

128. máma di ábizi, s.f. CA 'ape regina' - Vr209 ábi mámma Oristano 'ape regina'.

129. rénsa, s.f. CA 'farfalla parassita delle api' - SpanoII,320 renza 'lappa, insetto che ruba il miele', DES $(I, 104)$ arrèndza 'bruco della farfalla dell' arnia che distrugge i favi'

130. škursú́a, s.f. CF, škusúa CA, 'sciame d'api' - SpanoII,349 scussùra 'sciamo', Vr210 skussúra, DES $(\mathrm{I}, 667)$ iskussúra; concorre occasionalmente la forma lig. šámu, di uso corrente per indicare un gruppo di animali di grossa taglia (gregge, mandria) o di persone; cfr. anche il derivato škusuá 'sciamare'.

\section{j) Pesci e pesca. Lessico marinaro e di tonnara}

Sulla pratica della pesca presso le comunità tabarchine si veda quanto esposto nei paragrafi precedenti. Una parte consistente della terminologia relativa è d'origine sarda, importata dai pescatori algheresi che, scendendo lungo le coste occidentali della Sardegna, diedero un contributo essenziale al rilancio dell'attività ittica a Carloforte e a Calasetta. Un altro apporto determinante fu quello meridionale italiano, legato in parte alla pratica della tonnara, ambito nel cui lessico più sono frequenti i sicilianismi che le voci d'origine sarda.

131. bazúku, s.m. TB 'pagello occhialone' e spesso, in senso fig. 'ingenuo, stupido' - Ls214, DES $(\mathrm{I}, 186)$ bașíku 'pagello' (< cat. besúc, basúc); a CA è completamente sconosciuta la forma lig. bez $\overline{\tilde{u}} g u$, che a CF concorre col tipo sardo, ma soprattutto nel senso fig.

132. ćíu, ćiétu, s.m. TB 'tipo di barca a fondo piatto in uso nel tratto lagunare prospiciente Sant'Antioco e nei bassi fondali'; era consuetudine far trovare una di queste imbarcazioni davanti alla porta delle ragazze che avevano rifiutato un corteggiatore, donde la locuz. areštå $\eta$ šú $c$. 'rimanere zitella' - Spanol,332 ciu 'specie di barca'.

133. dugắle, drugắle, s.m. CF 'fune che lega la corda del tonno all' appiccatoio nello stabilimento a terra della tonnara' - Spano I,406 dugàli 'laccio, capestro'; Ls196, DES (I,482) dugáli 'laccio col quale il carnefice strozzava il condannato alla forca' poi 'cappio in genere'.

134. garẹ́tu, s.m. TB 'zerro' - SpanoII,57 giarrèttu; Ls214, DES (I,603) ğarréttu (< cat. gerret, xerret). 
135. kañíja, s.f. TB, kanína CF 'orata' - SpanoI,302 canina, DES (I,283) kanína ${ }^{68}$.

136. kọ́ćula, s.f. TB 'conchiglietta', 'arsella' - Spanol,335 còcciula 'chiocciola, arsella', DES $(1,359)$ kó$\check{c} \breve{c} u l a$; normalmente vengono pescate nella laguna di fronte a Sant'Antioco da raccoglitori locali.

137. levižéda, s.f. CF 'levicella della tonnara' - Assente nei repertori sardi consultati.

138. maćọ́ni, s.m. CA 'ghiozzo' - SpanoII,200 macciòni; Ls214 maččòni (< cat. maxón); a CF si usa il tipo lig. giǵún.

139. marićáda, s.f. TB 'mareggiata' - Forma che riflette la fonetica sulcitana, in concorrenza col tipo lig. maygásu.

140. marićọlu, s.m. TB 'maricino'; voce d'uso - Forma che riflette la fonetica sulcitana rispetto al sinonimo mayžá di tipo lig.

141. paǵẹ́lu, s.m. TB 'pagello, fragolino' - SpanoII,256; Ls215, DES (II,205) pağéllu (< cat. pagell o voce it.); concorre a CA la forma lig. pắgu.

142. palắya, s.m. TB 'sogliola' - SpanoI,256; Ls215, DES (II,207) paláya (<cat. pelaya o voce it. merid.).

143. rísa, s.f. TB 'rete', intendendo precipuamente quella da pesca, ma a CA anche rísa du létu 'rete del letto' - SpanoII,226 rèzza, DES (II,358); il tipo lig. rẹ́ è completamente sconosciuto ${ }^{69}$.

143. rokalẹ́du, s.m. CF 'tordo fischietto' - SpanoII,328 rocàle 'tordo di mare', logud., DES (I,127) arrokkáli, campid.

144. škríta, s.m. TB 'tipo di razza' - SpanoII,347 scrìtta; Ls215, DES (I,395) skrítta 'razza' (< cat. escrita); concorre ferása 'razza in genere', che è voce d'origine cat. presente sia in sardo che in lig. (VPL LSII,1, s.v.)

145. surẹ́lu, s.m. TB 'suro'; voce di uso corrente - Ls215, DES $(I I, 449)$ suréllu 'specie di scombro' ( $<$ cat. surell, sorell).

146. tạkadú, s.m. CF 'cerchietto per la nassa' - SpanoII,384 tancadùra, DES (II,463) tankadúra 'serratura'.

k) Pesi e misure, moneta, amministrazione

147. kaǵắize, $s . m$. TB 'moneta da pochi centesimi', nella locuz. u nu va $\eta$ $k$. 'non vale nulla', d'uso corrente - Spanol,294 cagliaresu 'la sesta parte del soldo sardo'; cfr. il nome di Cagliari in Tb, che è Káǵay a CF e Káǵe a CA.

148. muštazáfu, s.m. CF 'antica guardia municipale', voce non più usata se non in senso scherzoso - SpanoII,235 mustazzàffu; Ls194, DES (II,147) mustattsáffu 'ufficiale della grascia' (< sp. ant. almutazafe, ar. al-mohtasib).

149. regadú, s.m. TB 'dirigente, direttore', ma usato quasi esclusivamente per 'direttore, organizzatore di una festa' - SpanolI,317 reggidòri, Ls194, DES (II,347) reğğidòri 'consigliere comunale'

150. rumána, s.m. CA 'stadera'; non frequente rispetto al tipo lig. kaytá, d'uso corrente a CF SpanoII,329, DES (II,362) romàna.

151. ryá, s.m. TB 'moneta da pochi spiccioli' - SpanoI,199 arriàli; Ls208 arríális 'monete da due centesimi', cfr. DES (II,341) arriali, s.v. reále. 


\section{1) Arti e mestieri. Attrezzi di lavoro}

Si riuniscono qui voci non specificamente settoriali, ma penetrate nell'uso generale o come tecnicismi legati ad alcune professioni.

152. byarọ́ne, s.m. TB 'cemento allungato con poca sabbia' - SpanoI,263 biaròne 'calcina sciolta, allungata', cfr. DES $(\mathrm{I}, 205)$ s.v. bieròne, biaròne.

153. gabelọ́tu, s.m. TB 'tabaccaio' e 'tabaccheria', voce uscita dall'uso - Spanoll,50 gabellòttu 'tabacchìno', DES (I,564) gabelóttu. Concorre, oltre al più recente tabakín, anche il tipo štágku, largamente diffuso sia in Liguria che in Sardegna (cfr. nota 43).

154. konćakonkọ́ne, konǵagongọ́ne, kuyćapiñáte, s.m. CA 'ricucitore di conche, di pentole'; voci uscite dall'uso - Voci certamente sarde nel loro primo componente: cfr. Spanol,96 acconciacardàxius, 345 concialapòlu, acconcia cardagiu, DES $(\mathrm{I}, 371)$ akkontsalapiólu; è probabile che gli ambulanti sardi che praticavano questo mestiere adattassero $\mathrm{i}$ loro gridi di richiamo alla realtà linguistica tabarchina presentandosi come 'acconcia-conche' o 'acconcia-pentole', e che le voci così modificate penetrassero nel lessico locale.

155. palíta, s.m. TB 'paletta' in genere, e particolarmente 'paia del forno'; CF anche 'pala dei fichi d'india'; CA anche 'raschiatoio per l'aratro' - SpanoII,257; Vr104,159, DES (II,205) s.v. pala. Denominazione entrata nell'uso generale in virtù delle molte specializzazioni nelle quali era più probabile l'assunzione del prestito sardo.

156. prẹ́ysa, s.f. CF 'pressa'; a CA solo nella locuz. tẹ na bẹ́la p. 'sei un furbacchione'; non usuale - SpanoII,293, DES (II,307) prènsa 'strettoio, torchio'.

157. škaráda, s.f. TB solo nella locuz. $a$ š. 'a cottimo' - SpanoII,341 scaràda 'cottimo', DES (II,390) skaráda.

158. špadín, s.m. CF 'mannaia del macellaio' - SpanolI,363 spadinu 'coltellaccio'.

159. verdugílu CF, verdugrilu CA, s.m. 'sega a due mani' - SpanoII,414 verdughigliu; DES (II,571) verdugil' $u$, id. e Ls218 burdugil'u 'sega lunga' ( $<$ sp. verduguillo) a Cagliari.

\section{m) Parti della casa. Oggetti di casa, suppellettili, masserizie, utensili vari ecc.}

Si segnala in quest'ambito l'abbondanza di denominazioni relative a recipienti, sia in terracotta che di vegetali intrecciati. Tali produzioni provenivano in larga parte dal retroterra (le terraglie, in realtà, anche da Livorno e da Tunisi fino ai primi decenni del sec. XX), e ciò spiega la frequenza dell'assunzione di sardismi.

160. barabán, s.m. CA 'soppalco nelle case di campagna' - Sembra la corruzione della voce logud. barandàu Spanol,247 'pianerottolo', DES (I,176) barandáu 'ringhiera, parapetto', non senza richiami a una forma onomatopeica che evoca il rumore dello scalpiccio su un soppalco di legno.

161. bọ́vida, s.m. CA 'archivolto' - SpanoI,275, DES (I,221) bòveda 'volta'.

162. bukẹ́tu, s.m. CA 'mazzo di fiori'; voce inusuale - Spanol,281 bucchèttu: cfr. anche DETroRI, 1988: 304.

163. ćiminệa, s.m. TB 'piano di lavoro della cucina, focolare' - SpanoII,423 ziminèa, DES (II,591) tsiminèa 'camino'.

164. gwantẹ́a, s.m. TB 'vassoio, piatto da portata' - SpanoII,68 guantèra. 
165. kadín, s.m. TB 'canestro piatto, spesso utilizzato per il pesce' - SpanoI,293 cadinu 'vaso di legno a doghe'; Vr127, DES (I,259) kadínu 'cesto', 'cestone'.

166. kaníštru, s.m. TB 'graticcio, stuoia di canne' - Utilizzato solo in questo specifico significato, e soprattutto per il 'canniccio dei soffitti': cfr. DES $(I, 238)$ kanístru 'canestro'.

167. kartálu, s.m. TB 'paniere, cestino' - SpanolI,341 scartèddu; Ls143, DES (I,654) (i)skartéddu; L'adeguamento $-d d$ - $>-l l$ - potrebbe denunciare un'antichità del prestito o una provenienza di esso dall' area logud., a meno che non si tratti, addirittura, di una voce ital. merid. penetrata attraverso il lessico della pesca.

168. káša, s.f. CA 'cassapanca' - Spanol,314; Vr319 káša 'cassapanca'; la voce, foneticamente lig., significa in primo luogo 'cassa' in senso generico; a CF è in uso kašabánka, calco sulla voce it.; completamente assente il tipo lig. $u$ bajk $\overline{\bar{a}}$ che ha assonanze nel sardo logud. bangàle 'cassa grande', Spanol,247.

169. kátre, s.m. CA 'letto a una piazza di elegante lavorazione'; voce uscita dall'uso - SpanoI,317 catre 'letto di parata', DES (I,319) kátre 'letto di parata'.

170. lẹ́pa, s.m. TB 'scorticatoio, coltellaccio', in particolare (ma non solo) quello dei pastori - Spano II,188 lèpa, DES (II,22) lèppa.

171. mućọ́ni, s.m. CA 'pezzetti di brace'; voce uscita dall'uso - SpanoII,229 mrucciòni 'tizzone', DES (II,153) muttsịioni 'tizzone' (s.v. múttsu).

172. paraštáǵu, s.m. TB 'scaffalatura', sia di casa che di negozio - SpanoII,260 parastàggiu 'guardaroba, scaffale'; Ls212, DES (I,222) parastáğğu 'scaffale' (< cat. parastatge).

173. safáta, s.f. TB 'vassoio' - SpanoII,334 saffàta; Ls190,211, DES (II,376) saffátta (< cat. safata).

174. serî̌žu, s.m. CF 'crivello, piccolo vaglio' - Spanol,321 'vaglio, canestro grande', logud.; Vr153, DES (I,330) čerrìgu s.v. kerrikru.

175. šivẹ́la, s.f. TB 'conca, catino di terra impiegato per preparazioni alimentari e usato anche come piatto da portata' - SpanoII,345 scivèdda 'catino'; Vr154,155, DES (II,658) šivèdḍa 'bacinella in terracotta in cui si lavano le stoviglie' (s.v. iskívu); a Sant'Antioco. šiféḍ̣a (nostre informazioni); si noti - $l l$ - in luogo del consueto $-d d-<-d \underline{d}-$ -

176. špídu, s.m. TB 'spiedo' - Vr328 logud. ispidu.

177. štrệ́žu, s.m. TB 'recipente in genere' - SpanoII,371 strèxu de cuxina 'stoviglia', DES (II,691) stréžu s.v. istérịu.

178. štućadinái, s.m. CF, štuǵadinấi CA 'salvadanaio' - SpanoII,372 stuggiai 'conservare'.

\section{n) Abbigliamento e acconciatura}

Presso le comunità tabarchine non esistette mai un costume tipico, ed era anzi un segno di distinzione rispetto ai Sardi il vestire abiti di foggia continentale: «[Alla sagra di Sant'Antioco] si girava curiosi verso le esposizioni delle varie bancarelle in mezzo ad una gran folla in costume sardo che era il quotidiano abito di quegli abitanti; noi non distinguevamo certamente il costume feriale da quello festivo. Erano tutti vestiti alla sarda e questo distingueva i Calasettani ed i Carlofortini da loro» 70 .

179. arbắžu, s.m. TB 'orbace' - voce conosciuta principalmente in relazione all'abbigliamento tipico sardo: SpanoII,249 orbàci; Vr292, DES $(\mathbf{I}, 68)$ orbáči, arbáči s.v. albáke. 
180. bọ́ti, s.m. TB 'stivaloni' - SpanoI,274 bottìnu 'stivaletto'; Ls203,219, DES (I,220) bòttas 'stivali' (<sp. botas).

181. kanáka, s.f. TB 'collana' - Spanol,303 cannàca 'collana, monile, vezzo'; Vr313, DES $(I, 284)$ kannákka, Ls253.

182. mantẹ́ka, s.m. TB 'lucido da scarpe'; voce d'uso - SpanoII,208, DES (II,68) mantèga 'panna conservata', e mantegil'a 'pomata'; in Liguria la voce è documentata dal sec. XVIII (Toso, $1993,94)$, ma non in questo specifico significato.

183. pyúnku, s.m. CF 'pedule'; voce rara rispetto al sinon. škapín, di tipo lig., d'uso corrente anche a CA - SpanoII,274 peùncu, DES (II,256) peúnku.

184. šíptu, s.m. TB 'cinta dei pantaloni'. SpanoI,330 cìntu, DES (I,341) číntu, su zíntu s.v. kíntu; concorrono (più diffuse) le voci lig. kurẹ́za, sentǘa.

\section{o) Cibi e loro preparazione}

Di particolare rilievo in questa sezione sono i nomi dei dolci, che presso le comunità tabarchine sono quasi tutti di tipo sardo; discreto interesse ha anche la nomenclatura legata alla panificazione.

185. birikíti, s.m. pl. CF 'dolcetti fritti di pasta, olio e zucchero'; voce d'uso - pirikíttus VI176, DES (II,275) 'dolcetti globosi di pasta all'uovo'; Ls208; SpanoII,280 pirichittus 'zuccherini'.

186. gatọ, s.m. TB 'dolce di mandorle tostate' - SpanoII,52, DES (I,572) gattò; cfr. anche DETTORI, 1988: 298.

187. gếrdye, s.f. pl. CF 'pezzetti di sugna che avanzano dalla produzione dello strutto'; voce uscita dall'uso - SpanoII,54 gerda 'cicciolo'.

188. ǵ́ǵeri, s.m. pl. TB 'dadini di pasta fritti e caramellati' - Spanol,333 cìxiri 'ceci': il passaggio semantico si spiega con l'aspetto di questi piccoli dolci.

189. inkažấu, agg. CF 'condito col formaggio' - SpanolI,94 incasài 'asperger con cacio'.

190. kasọ́la, s.f. TB 'zuppa di pesce' - SpanoI,315 cassòla 'guazzetto, umido, stufato'; Ls209, DES (I,314) kassòla 'specie di umido fritto in casseruola, così con carne come con pesce' e 'guazzetto di pesci' (< cat. cassola).

191. kaškẹ̉te, s.f. pl. CA 'dolce con mosto cotto e semola' - SpanoI,314 caschètta 'ciambella'; kaskètta Vr177, DES (1,312) 'sorta di ciambella';

192. kraká, v. tr. TB 'impastare in pane coi piedi' - Spanol,361 craccài; Vr267 krakkare 'calcare', DES (II,297) krakkái s.v. karka.

193. kukội, s.m.pl. CA 'un formato di pane'; cfr. anche il dimin. kukuẹ́di 'pezzi di pasta di pane cotti a parte per i bimbi' - Spanol,335 coccòi; Vr167, DES (I,355) kokkòi 'tipo di pane' s.v. kòkka 'pane a ciambella'

194. maravílye, s.f. pl. TB 'fritture di pasta dolce, tipiche dei rituali di comparaggio che si svolgono durante la notte di San Giovanni Battista' - Vr174 maravil'as, DES (II,72) maravil'a.

195. mása, s.f. CA 'interiora, rigaglie del pollame'; la voce circola anche l'espressione ibrida nu te ne

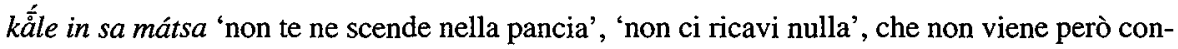
siderata squisitamente tabarchina (si noti infatti l'utilizzo dell' articolo sardo e l'aspetto fonetico mantenuto in questo caso dalla voce) - SpanoII,214 mazza 'budella, interame, intestini' . 
196. papasína, s.f. TB 'pina d'uva passa', dolce tipico - SpanoII,255 pabassinu; Vr175 pabassínas, DES (II,215) pabassínu s.v. papássa.

197. sắba, s.f. TB 'mosto cotto'; voce entrata anche nell'it. locale - SpanoII,333 sàba; Vr175, DES (II,382) sába 'sapa' s.v. sápa.

198. sufrăža, s.f. TB 'focaccia di cruscherello', anche nella forma dimin. sufražéta - SpanoI,333 civràxu, Vr146, DES (I,351) čivrážu 'cruschello' s.v. kivárdzu.

199. sukítu, s.m. TB solo nella locuz. a s. 'in umido' - SpanoII,375 succhètte, succhittu, DES (II,439) sukkètte, sukkíttu 'guazzetto' s.v. súkku.

200. šápidu, agg. TB 'insipido, scipito'; anche in senso fig. 'persona insignificante', 'priva di carattere' - Voce d'uso, ma meno diffusa della forma lig. fátu; SpanoII,344 sciapidài 'fare scipito' (il tipo desápitu non è sconosciuto neppure nell'estrema Liguria orientale, VPL).

201. šípure, s.f.pl. CA 'zippole' - SpanoII,423 zipula, Vr174 tsíppulas 'specie di frittella tonda'; tipo assente in Liguria.

202. štínku, s.m. CA 'olio di lentischio, un tempo preparato artigianalmente e usato in sostituzione dell'olio d'oliva'; voce uscita dall'uso - Deriverà da stìngu 'sottile' (SpanoII,370), in quanto si trattava di un olio molto leggero.

203. tinbála, s.f. CF 'timballo' - SpanoII,391 timbàla, DES (II,484) timbálla 'forma per maccheroni o polenta' e 'pasticcio di maccheroni'.

p) Lessico infantile. Giochi e svaghi

204. bábu, s.m. TB 'babbo', 'padre'; anche (ba)bugránde 'nonno', tipo quest'ultimo di impianto lig. (pwegrá́nde) - SpanoI,240, DES (I,162) bábbu ${ }^{71}$.

205. baralíku, s.m. TB 'girlo, gioco del girlo' - SpanoI,247 baraliccu, DES (I,176) bar(r)alikku.

206. ćućú, s.m. TB nella locuz. a ć. 'a passeggio' - SpanoI,332 andai ciucìu 'andare a spasso', e cfr. DES $(I, 453)$ čiččíu 'beniamino di casa'

207. gwadufọ́rti, kwádufộrti, s.m. CA 'scavalcata in serie', letteralmente 'cavallo forte' - a CF kavalása.

208. kabẹ́su, s.m. CA 'scapaccione dato ai ragazzi' - Spanol,289 cabèssu 'schiaffo', DES (I,252) kabéssu s.v. kabessáda.

209. kunkẹ́da, s.m. CF 'gioco del rincorrersi'; voce pressoché uscita dall'uso.

210. šanpitá, v. intr. CA 'far festa con canti e balli' - SpanoII,344 sciampittài 'scambiettare, far scambietti', sciampitta 'salto nel ballo, capriola', DES (II,454) šampittai.

211. štrúnpa, s.f. CA 'un gioco di abilità'; voce uscita dall'uso e comunque attribuita esclusivamente ai carradori sardi, che praticavano questo gioco nei periodi di riposo durante la vendemmia SpanoII,372 pigai a strumpas 'lottare', DES $(1,700)$ istrumpare 'gettare a terra'.

q) Determinazioni di qualità, carattere, provenienza riferite a persona

212. aškeúzu, agg. CA, škeúzu CF 'eccessivamente scherzoso' - Spanol,207 ascheròsu 'nauseante' (sp. asqueroso).

213. azuríu, agg. CF 'tirchio' e 'avido' - SpanoI,218 asùridu 'avido, ingordo'. 
214. barộzu, agg. TB 'seccatore, importuno, millantatore'; a CA anche 'nomignolo usato per indicare gli abitanti di Sant'Antioco' - SpanoI,251 barròsu logud. 'loquace, millantatore', DES (I,181) s.v. bárra.

215. bibínku, agg. CF, pibínku CA 'tirchio' a CF; 'sofisticato', 'eccessivamente lezioso', CA SpanoII,275 pibincòsu 'seccante, noioso', DES (II,258) pibinka 'seccaggine', pibinkai 'annoiarsi', s.v. pibiare.

216. famigộzu, agg. CF 'avido, famelico' - Spanoll,20 famigòsu 'misero, affamato', DES (I,502) famigóșu.

217. feminẹ́da, s.m. CF 'individuo effemminato'.

218. malintráña, agg. CA, målazintráña CF 'cattiva disposizione dell'animo' a CA; CF 'musoneria' e 'musone'; non comune - SpanoII,124 homine mal'intragnadu 'sleale, pessimo, di cattivo cuore', DES (II,640) malintrañádu s.v. intráñas.

219. ōgyắnu, agg. TB 'vorace, goloso' - SpanoII,247 ojànu 'ingordo', DES (II,183) ogiánu s.v. ókru. 220. pếgu, s.m. CA 'tipo losco' - DES (II,238) pégus nel senso fig. di 'cattivo individuo' (cfr. it. buonalana), specifico del campid.

221. šperdisyå, v. tr. TB 'dissipare' - Cfr. la voce seguente.

222. šperdisyấu, agg. TB 'dissipatore, persona disordinata nei suoi interessi' - Spanoll,365 sperdiziai 'sperperare', DES (I,676) isperdítssiu 'sperpero'.

223. štránću, agg. CF, štránǵu CA 'straniero' e 'estraneo' - Si preferisce la forma lig. fueštẹ́; Spano II,371 stràngiu 'straniero', DES (II,696) stránğu s.v. istrándzu.

r) Stato fisico; salute e malattie

224. asidî́u, agg. CA, sidî́u CF 'intirizzito' e 'impalato' - SpanoII,356 siddìu 'intirizzito', DES (II, 416) sị̣dai is dèntis 'stringere i denti' s.v. siḍ̣ai 'stringere'.

225. bašitẹ́du, agg. CF 'piuttosto basso'.

226. ćúrpu, agg. CA 'guercio' e anche 'cieco' - SpanoII,426 zùrpu 'cieco', DES (II,555) tsúrpu s.v. qúrpu; Vengono frequentemente confuse le voci che significano 'guercio', 'cieco' (gwếrsu) e 'strabico'.

227. fugẳle, s.m. CF 'irritazione all'ugola, alla gola' - SpanoII,34 fogàle 'malattia dei porci, infiammazione alle ghiandole'; Vr259, DES (I,529) fogáli 'infiammazione delle ghiandole del collo' e 'angina del maiale', s.v. fokále.

228. kaleptû́ra, s.f. CF 'eccitazione febbrile'; voce rara, a CA conosciuta come sarda ma non usata - SpanoI,296 'febbre'; Ls221, DES (I,269) kal(l)entúra 'febbre'.

229. pilardẹ́da, s.f. CF 'un malanno'; voce rara, registrata solo nella locuz. ke me veñíse a p. 'mi venisse un accidente!' - SpanoII,278 pilardèdda 'noce vomica', da pilárda 'pera secca', DES (II, 125).

230. pištinká, v. intr. CA 'essere balbuziente'; voce rara - SpanolI,283 pistincare 'scoccare il dito medio dal pollice per battere', logud., DES (II,282) pistínku 'buffetto' (s.v. pistònka). 


\section{s) Religione e ambito religioso}

231. inkúntru, s.m. CA 'cerimonia religiosa dell'encuentro durante la quale si raffigura, durante il Venerdì Santo, l'incontro tra il simulacro della Vergine e quello di Gesù' - Ls197, DES (I,625) $s^{\prime}$ inkóntru ${ }^{72}$.

232. matráka, s.f. CA 'battola, raganella della Settimana Santa' - SpanoII,213 matràcca; Ls200, DES (II,88) matrákka 'tabelle della Settimana Santa'. A CF solo il tipo lig. batáila.

233. šfẹ̃ra, s.f. CF (ALI) 'ostensorio' - In questo significato, voce menzionata dallo Spano nella sezione Italiano-Sardo, II,157 (isfera) e DES (I,669) isfêra.

t). Lessico espressivo

234. aćirá, v. tr. TB 'afferrarsi, abbrancarsi' - Spanol,94 accirrài 'afferrare', DES (I,343) s.v. kírra.

235. aǵutộryu, s.m. TB 'aiuto', solo in contesti scherzosi o espressivi - SpanoI,122 aggiutòriu 'aiuto, ausilio'; Ls238 ağğitórinu 'aiuto', DES (I,63) ăğğut(t)órịu.

236. āmarọ́la, avv. TB 'malvolentieri', 'a malincuore' - SpanoI,142 amarolla 'di mala voglia'.

237. arōšá, v. tr. CA, arušá CF 'infastidire' - SpanoI,202 arròsciri 'annoiare, tediare'.

238. atuntấu, agg. CF 'istupidito' - SpanoI, attontadu 'stupido, insensato', DES attontádu (II,496) s.v. tóntu.

239. āvulọtu, s.m. TB 'baccano, chiasso, confusione' - SpanoI,233, DES (1,69) avolotu 'scompiglio, tumulto' s.v. alborot(t)ai.

240. avulutấ, $v$. intr. TB 'schiamazzare' - Spanol,233 avolotài 'turbare, scompigliare'.

241. ažíu, s.m. CA solo nella locuz. u g á a. 'hai voglia!' - A Sant'Antioco ténniži ažíu 'hai aceto!' nello stesso sign.

242. besída, s.f. CF 'battuta, motto di spirito' - SpanoI,261 'uscita'.

243. budíku, s.m. TB 'pasticcio' - SpanoII,79 imboddicu 'imbroglio, intrigo, confusione', DES (I,615) s.v. imbolikare.

244. burupbála, s.f. CF 'scompiglio' - Spanol;286 logud. burrumbàglia 'confusione, disordine, strepito', DES (I,245) burrumbálla.

245. filumệ́u, inter. CA 'caro mio', intercalare soprattutto femminile, di uso estremamente episodico - Da fillu méu.

246. fứi fứi, avv. TB 'detto di persona che fugge' - SpanoII,45 istare fui fui 'fuggiachiare'.

247. gargalọ́ne, s.m. CF 'giovincello petulante' - Probabilmente da gregáğu 'capretto', DES (I,589) s.v. gangádzu, voce introdotta nel Campidano nella forma gragállu attestata (cfr. anche SpanoII,63; Vr254), e di qui in TB con metatesi.

248. inbrušiná, igbušiná, v. tr. TB 'avvolgere in maniera sommaria e disordinata' - SpanoII,80 imbruscinài 'rivoltolare', DES $(\mathrm{I}, 616)$ imbrošinare 'ravvolgere'.

249. inbudikấ, v. intr. CF 'pasticciare, far pasticci' - SpanoIl,79 imboddicai 'avvolgere, avviluppare', DES (I,615) imboddikare 'mbrogliare' s.v. imbolikare.

250. inkankaráse, $v$. intr. pron. TB 'rimanere bloccato per un crampo, una fitta' - SpanoI,301 cancarài 'indolenzire', DES (I,281) kankarai s.v. kánkaru.

251. inkrabắu, agg. CA 'incantato, stupito' - SpanoII,99 incrabinare 'essere spiritato', DES (I,39798) inkrabinare s.v. krápa. 
252. kráštula, s.f. TB 'pettegola'; voce d'uso - Spanol,361 cràstula 'pettegola', crastulai 'spettegolare', DES $(\mathrm{I}, 400)$ krástula 73 .

253. ki d á kriấu, inter. CA 'chi ti ha creato' per mandare al diavolo una persona e i suoi genitori Elemento fraseologico non assimilato, ritenuto estraneo al tabarchino ${ }^{74}$.

254. malápa, inter. TB 'imprecazione stizzosa', anche nella locuz. m. aw pekáu 'maledizione al peccato' - Sardo málu áppa, come it. merid. mannaggia.

255. murigá, v. intr. TB 'rimenare, rimestare' - Vr268 murigai 'rimestare', DES (II,139) s.v. murikare.

256. papadộri, s.m. CF 'mangione'; usato soltanto in contesti scherzosi - SpanoII,259 pappài 'mangiare', DES (II,219) s.v. pappare.

257. rộš̌a, s.f. CF 'fastidio, briga' - Spanol,202 arròsciu 'annoiato, seccato', DES (I,128) arròširi.

258. rudắ, v. intr. CF 'bighellonare, gironzolare' - Spanol,201 arrodare 'andar in giro'.

259. sapulắse, $v$. intr. pron. CF 'strapazzarsi'; a CA atsapulắse 'arrangiarsela' non è considerata voce del dialetto (come del resto denuncia chiaramente la fonetica), ma si tratta di una parola conosciuta, utilizzata in contesti scherzosi e gergali - SpanoI,237 azzapulài 'agitarsi, sbattersi', DES (I,144) attsappulai 'sbattere, scuotersi', s.v. attappare.

260. šimínǵu, s.m. TB 'pensiero fisso' - SpanoII,344 scimingiu 'sbalordimento, stordimento', DES $(\mathrm{I}, 555)$ šumínğu, šimínğu 'sbalordimento', 'capogiro', s.v. fúmu.

261. škramyå, v. intr. CA 'miagolare', ma soprattutto 'imprecare' - Spanoll,347 scramiài 'gridare', DES $(1,397)$ kramai s.v. kramare.

262. škutulá, v. $t r$ TB 'scuotere' anche in senso fig. per 'picchiare, dare una lezione', da cui il deriv. škutuláda 'ramanzina' - SpanoII,349 scutulai.

263. špramá, v. $t r$. CF 'anelare, desiderare ardentemente'; voce d'uso; špramáa $\mathrm{CA}$ 'di colore intenso' - SpanoII,367, DES (I,681) ispram(m)are, spram(m)ai 'spaventare'.

264. traypáya, s.f. TB 'frode, inganno' - SpanoII,396, DES (II,505) trámpa 'frode, tranello', ma cfr. le osservazioni alla nota 43.

265. traypúzu, agg. CF 'ingannatore' - SpanolI,396 trampòsu, DES (II,505) trampóșu 'truffamondo's.v. trámpa.

u) Varia

266. akansá, v. tr. TB 'guadagnare, ottenere' - SpanoI,89 accansàre, I,129 alcanzài 'conseguire, perpetrare, ottenere', DES (I,69) akkantsai s.v. alkansare, akkansare.

267. akọ́su, s.m. CF 'punto d'appoggio' - SpanoI,99 accòzzu 'appoggio, sostegno', DES (I,393) akkottsai, akkóttsu 'appoggio' s.v. kò $\theta \theta a$.

268. amuntumắ, v. tr. TB 'ammucchiare' - Spanol,152 ammuntonàre.

269. apažá, v. tr. CF 'pacificare' - SpanoI,173 appaxiài 'pacificare'; Vr264 appașare 'dividere in modo equo', Marghine, DES (II,202) appažiai s.v. páke.

270. ažbašå, v. tr. TB 'scendere' e anche 'uscir di casa' - Questo particolare uso del verbo lig. sembra un calco semantico su campid. abbašái 'discendere'.

271. dezíǵu, s.m. CF, dizíǵ $u$ CA 'desiderio'; voce usata per lo più in senso ironico - SpanoI,395 disigiu, DES (I,471) dișíğğu s.v. dișidzare. 
272. gána, s.f. TB 'voglia', per lo più in espressioni come $u$ lẹ de mála gána 'non ne ha voglia'; di uso raro rispetto alla forma lig. kwẹ - Spanoll,51, DES (1,568) gana 'voglia'; a mala gana 'a cattiva voglia'.

273. kwitá, v. intr. CF, koytả CA 'camminare in fretta' - Spanol,368 cuidài 'far presto', DES (I,363) koitare(sì), akkoitai(și) 'affrettarsi'; il tipo kúita 'fretta' è presente nella Liguria occidentale (VPL s.v. cùita).

274. ordinǵá, v. tr. TB 'allestire, preparare' e fig. 'conciare per le feste' - SpanoII,249 ordingiài, DES $(\mathrm{I}, 191)$ ordindzare, ordinğai 'allestire'.

275. pasadísu, s.m. TB 'vicoletto, corridoio tra le case'; indica anche 'l'istmo che congiunge l'isola di Sant'Antioco alla Sardegna' - SpanoII,263 passadissu, DES (II,230) passadíssu 'passatoio, andito, androne'; Ls212 passadíssu 'corridoio'.

276. šauprá, v. tr. CF 'allargare, dilatare' - SpanoI,344 sciamplài 'allargare, dilatare'.

277. šinbulấ, v. tr. TB 'disturbare' a CF; 'svegliarsi presto' e anche 'spicciarsi, sbrigarsi' CA, CF SpanoII,345 sciumbullài 'diguazzare, intorbidare'; Vr250, DES (II,446) šumbullai 'sconvolgere' s.v. supudzare; šumbúlla! 'alzati!'; la -i-della voce TB potrebbe presupporre un precedente $-u$ - > $-\ddot{u}$ - che confermerebbe l'antichità del prestito.

278. škramentắ, v. tr. CF 'scottare' - SpanoII,347 scramentài 'scottare', DES (I,653) iskarmentare, skramentai 'imparare a proprie spese'.

9. Un aspetto importante della problematica legata all'assunzione di voci d'origine sarda in tabarchino è quello relativo alla percezione di tali fenomeni di prestito da parte dei parlanti.

L'alterità delle due comunità tabarchine rispetto al retroterra è stata vissuta in passato, come si è detto, in relazione a una profonda diversità di specializzazioni economiche: è per questo che la locuzione aná in Sardẹña 'andare in Sardegna', con la quale i Tabarchini intendono tuttora 'andare in qualsiasi località sarda', compresa la vicinissima Sant'Antioco, assume un valore che trascende completamente ogni considerazione di carattere etnico.

Questa precisa consapevolezza di un'alterità economica si appoggia peraltro agli elementi distintivi di carattere linguistico e in senso lato culturale, che, per la loro stessa evidenza, diventano i facili blasoni di una «genovesità» conclamata e per certi aspetti consapevolmente ristrutturata in alcuni dei suoi caratteri più vistosi ${ }^{75}$.

Il concetto-base della costruzione identitaria tabarchina viene così riassunto dal detto

se vágu pe má i tư̆̉rki $\mathrm{m}$ aćápay, se vágu pe tệ́ra i sắrdi $\mathrm{m}$ amása ${ }^{76}$,

nel quale è palese l'impegno di enfatizzare una differenza rispetto agli abitanti del retroterra, i Sắrdi 'i Sardi', paragonandoli addirittura agli antichi nemici, i corsari barbareschi ${ }^{77}$.

Come è dunque evidente, i Tabarchini rifiutano esplicitamente di considerare se stessi come Sardi, anche se questa distinzione perde valore nel momento in cui una 
persona proveniente dalla Sardegna si integra in una delle due comunità, assimilandone in primo luogo gli usi linguistici ${ }^{78}$. Il prototipo del 'Sardo' per il quale si nutriva (e in parte tuttora si nutre) un senso di netto distacco è piuttostó il Sardọ́tu akurdấu il 'sardetto a stipendio' che si adattava a umili mansioni presso i proprietari o si metteva al servizio della borghesia imprenditoriale tabarchina ${ }^{79}$.

È a questo tipo di manodopera scarsamente qualificata, precaria, per certi aspetti seminomade, che sembra adattarsi in particolare la locuzione amía $k$ aćámu $w$ sấrdu 'bada che chiamo il Sardo', con la quale si minacciano i bambini vivaci, a rappresentare ciò che in italiano è correntemente 'l'uomo del sacco' o altra forma di spauracchio. Anche altre locuzioni quali ẹ́ kộ́se da sắrdi 'sono porcherie, è spazzatura' (anche dumán ến kốse da sắrdi 'domani sarà un disastro, saranno cose turche'), e víve da $s a \dot{r} r d u$ 'fare il fannullone', chiariscono meglio il senso di una contrapposizione legata piuttosto alle categorie urbano $\sim$ rurale (pastorale) o stabile/accentrato $\sim$ seminomade/ disperso che non a una categorizzazione di carattere etnico ${ }^{80}$.

10. Se si considera la valutazione fortemente positiva dell'uso del tabarchino, non sorprenderà allora che ad esso si attribuisca, rispetto all' apporto sardo, una impermeabilità e una purezza che appare ampiamente smentita dalla quantità di prestiti penetrati dal sardo. Alla domanda

ti dí $\mathrm{k}$ ọ́w sẳrdu u lá lašắu kwarkộ́sa ō tabarkíne?,

la prima risposta di un informatore calasettano cinquantenne colto, rappresentativa di un'opinione corrente, tende ad esempio a minimizzare:

nínte. škwệži nínte. ćoẹ g ẹ de páwle nta nộštra lệngwa ke certamẹ́nte sún d orígine sắrda. e áwa li pe lí nu me $\mathrm{n}$ aregộ́rdu mánk ú̉ya kuší kúme ešprešúne, nu sọ́, ánke kúme eškramasyún... ${ }^{81}$.

Indicativo di questa mentalità appare anche il giudizio di un cultore di cose calasettane, Giovanni Cabras, che in una sua operetta sul dialetto locale approda a una sorta di improbabile purismo tabarchino: «Dobbiamo in ogni modo, riconoscere una nostra tara dovuta all'abusivo uso di parole del dialetto sardo incuneatesi nel nostro modo di parlare fin dai tempi lontani. È una grossa stonatura al nostro modo di esprimerci [...]. Sarebbe auspicabile una bonifica per eliminare questi suoni obrobriosi (sic!) dalla nostra parlata» ${ }^{82}$. Risulta allora quanto meno singolare che, in un repertorio di Antiche parole del dialetto calasettano annesso al suo lavoro, Cabras inserisca poi, con una certa abbondanza, voci di pretta provenienza sarda ${ }^{83}$.

In realtà, negare o minimizzare l'evidenza della componente sarda nel lessico tabarchino non significa dunque promuoverne (al di là di velleitari proclami) la sistematica espunzione, o attuarne la rimozione dall' uso mediante la pratica di assurde forme di autodisciplina: significa in fondo evidenziare, sia pure a livello inconscio, l'effettiva funzione storica e la reale portata sociolinguistica di tale apporto, che è quella 
di un arricchimento lessicale necessario e inevitabile fin dalla fondazione delle comunità sardo-liguri, ma certo non tale da essere percepito come fattore dirompente, come elemento di crisi nella sostanziale fedeltà dei Tabarchini alle consuetudini linguistiche originarie.

I parlanti, del resto, rivelano spesso una discreta competenza nella valutazione dei singoli lessemi di provenienza sarda. Più di una volta, nel corso delle inchieste, mi è capitato che gli informatori sottolineassero con decisione ${ }^{84}$ l'origine sarda di una voce, riconoscendone anche le diverse modalità di radicamento nelle consuetudini locali ${ }^{85}$.

Anche a livello diffuso, quindi, la percezione del fenomeno di prestito come elemento di arricchimento del patrimonio linguistico sfuma in fondo, necessariamente, lo scenario di una conflittualità tra sistemi antitetici, ed evidenzia piuttosto, al tempo stesso, una consapevolezza dell'alterità linguistica tabarchina come fattore imprescindibile di una specificità vissuta senza sindromi da accerchiamento, aperta alla contaminazione e allo scambio appunto perché fortemente consapevole della forza della propria originalità.

\section{Note}

1 Bottiglioni, 1928: 74. L'interesse per il tabarchino da parte di studiosi appartenenti alla cosiddetta dialettologia «prescientifica» è stato segnalato da DETTORI, 1978-80.

2 Il genovese parlato nei centri di Carloforte e Calasetta nell'arcipelago sulcitano in Sardegna è noto come tabarchino in quanto le due località furono fondate nel sec. XVIII da coloni provenienti dall'isola tunisina di Tabarca, ove una comunità ligure risiedeva a partire dal sec. XVI. Un sintetico riassunto delle vicende storiche, con attenzione ai fatti linguistici, è ora in Toso, 2000a: 336-340.

3 Un'analoga valutazione vale comunque, a mio avviso, anche per situazioni nelle quali il contatto linguistico ha prodotto fenomeni più consistenti (e vistosi) di commistione lessicale: rimando in proposito a quanto osservato sul lessico d'origine ligure nella parlata còrsa dell'isola di Capraia in Toso, 1999.

4 SOBRERO, 1971, versione ridotta in SOBRERO, 1969; versione leggermente modificata SOBRERO, 1974.

5 SOBRERO 1974: 19.

6 SOBRERO 1974: 30. Le valutazioni dello studioso vanno del resto contestualizzate in una fase della riflessione dialettologica che nella prima metà deglia anni Settanta, attribuendo una posizione intrinsecamente debole alle eteroglossie interne, ne riteneva ormai imminente il cedimento di fronte al progredire dei processi di koinizzazione in atto. Su un'analoga posizione sostenuta da G. TROPEA, 1970, 31 relativa alla regressione del galloitalico di Sicilia, si vedano le osservazioni di V. ORIOLES, 1997, 11: «In verità dal 1970 ad oggi molte cose sono cambiate, dimostrando, qualora ce ne fosse bisogno, che non solo sui destini dell'umanità e sugli equilibri internazionali ma anche sulle sorti di una parlata è arduo formulare previsioni, dovendo qui fare i conti con una variabile che, da Weinreich in avanti, viene definita 'lealtà linguistica', ossia con l'orgoglio di una comunità di rivendicare la propria alterità linguistica».

7 Sulle altissime percentuali della dialettofonia nelle due comunità tabarchine si veda il recente saggio di SITZIA, 1998; su altri aspetti sociolinguistici, e in particolare sul prestigio del tabarchino presso i parlanti, Toso, in corso di stampa A.

8 «Ma l'economia delle due colonie ha una storia tutta particolare: per molto tempo fu legata esclusivamente (e lo è ancora precipuamente) al mare, mentre l'agricoltura fu intrapresa relativamente tardi e solo come attività complementare» (SOBRERO, 1974: 18). Un'analoga lettura viene proposta da BLASCO FERRER, 1994: 155: «L'attività tradizionale principale è certamente la pesca». In realtà Calasetta ha basato storicamente la propria economia sulla monocultura delle vite (fino agli anni Settanta del sec. XX), e comunque anche a Carloforte, come si vedrà più oltre, la preponderante attività marittimo-mercantile pose sempre in secondo 
piano la pesca. Le colonie nacquero del resto, nel sec. XVIII, per l'esigenza del governo sabaudo di assicurare la coltivazione dei terreni incolti dell'arcipelago sulcitano. Si vedano in proposito $i$ testi degli accordi di colonizzazione dell'isola di San Pietro (in Vallebona, 1988: 29-31) e di Sant'Antioco (Cabras - Rivano POMA, 1992: 15-18): la distribuzione dei terreni e il loro sfruttamento agricolo costituirono le condizioni essenziali per il consolidamento degli insediamenti.

9 SOBRERO, 1974: 19.

10 SOBRERO, 1974: 30.

11 Intorno alla specificità tabarchina e a quella delle altre eteroglossie interne in Italia si è recentemente riacceso il dibattito scientifico, che investe anche problemi contingenti come quello di una corretta applicazione delle norme di tutela delle minoranze etnico-linguistiche alla luce della nuova legge 482 (in applicazione dell'articolo 6 della Costituzione). La richiesta di una tutela per varietà linguistiche come il tabarchino e il galloitalico di Sicilia e di Lucania (le cosiddette «eteroglossie interne», è l'oggetto della mozione finale del convegno internazionale di studi Insularità linguistica e culturale. Il caso dei Tabarchini di Sardegna (Calasetta, 23-24 settembre 2000) che si leggerà nei Documenti del convegno stesso, in ORIOLES - Toso, in corso di stampa.

12 BLASCO FERRER, 1994.

13 Dello stesso autore si veda anche l'Introduzione (pp. VII-XXVII) a SITZIA, 1998. Sui problemi della collocazione del tabarchino nel contesto ligure cfr. anche Toso, in corso di stampa B.

14 Un'analisi del sistema cognominale delle due comunità rivela come la componente tabarchina sia lontana da rappresentare la maggioranza della popolazione di Carloforte e Calasetta. Oltre ai numerosi Liguri rivieraschi, non Tabarchini, stabilitisi nei due centri (soprattutto a Carloforte) al momento stesso della loro fondazione, notevole fu la successiva immigrazione di pescatori e marittimi campani (soprattutto Ponzesi) e siciliani, di Ragusei, di Livornesi, di tecnici minerari savoiardi e francesi e, naturalmente, di Sardi: questi incrementi di popolazione non hanno avuto conseguenze apprezzabili dal punto di vista linguistico, così come non ne ebbe il tentativo di trasferire a Calasetta, pochi anni dopo la sua fondazione, un contingente di famiglie piemontesi (per un certo periodo componente maggioritaria della popolazione), in gran parte rimpatriate per i contrasti coi Tabarchini e le difficoltà di ambientamento.

15 Carloforte viene fondata nel 1738 , Calasetta nel 1770 dagli ultimi Tabarchini residenti nella sede originaria e da altri che si erano trasferiti a Tunisi o in località della costa maghrebina, ove del resto, ancora per gran parte dell'Ottocento, la presenza tabarchina fu numerosa e diversificata (Toso, 2000a: 337-338).

16 È impossibile riassumere qui, anche per sommi capi, la sostanza delle vicende economiche legate al popolamento di Tabarca e ai successivi insediamenti sardo-liguri. Al di là delle motivazioni contingenti (l'aumentata pressione tunisina sull'unica comunità cristiana insediata sulle coste del Maghreb, in concomitanza con l'accresciuta presenza politico-militare francese), la diaspora tabarchina ebbe poi caratteri insoliti nel quadro dei ripopolamenti delle zone costiere della Sardegna incoraggiati dalla nuova amministrazione sabauda. Tabarca genovese aveva costituito per circa due secoli una importante realtà economico-commerciale, gestita da una popolazione che, al momento dei suo trasferimento, poteva contare su notevoli potenzialità e su capacità imprenditoriali, sostenute da capitali liguri che continuarono a supportare le nuove attività economiche dei Tabarchini - in particolare l'importantissima pesca del tonno -, favorendo anche una serie di relazioni con la Tunisia, dove, in particolare nel corso del sec. XIX, diverse attività erano in mano a imprenditori genovesi più o meno coinvolti nella gestione del regime beilicale prima, e del protettorato francese poi. La proprietà e l'appalto delle tonnare sarde e di quelle tunisine di Sidi Daud in particolare, gestite da manodopera ligure e tabarchina, fu in mano a operatori genovesi addirittura fino agli anni Cinquanta del sec. XX; Genova, Savona e La Spezia erano inoltre i porti di sbarco del minerale sulcitano imbarcato a Carloforte e del vino da taglio che per circa un secolo garantì la prosperità economica di Calasetta; genovesi erano le ditte di trasporti che gestivano nell'Ottocento i contatti tra Carloforte e Cagliari e tra Cagliari e Tunisi; Genova era ed è tuttora il porto d'imbarco per i marittimi carlofortini, che costituiscono una parte importante della popolazione attiva della comunità (sede non a caso di un Liceo Nautico). Non è da stupirsi quindi che Genova e la Liguria siano anche la meta tradizionale di una significativa emigrazione dai due centri sulcitani.

17

La conservatività del tabarchino rispetto al genovese comune, sostenuta dal Bottiglioni nel suo saggio, appare ampiamente ridimensionata alla luce della documentazione storica e all'analisi comparativa del tabarchino rispetto alle parlate liguri continentali odierne; emergono piuttosto tratti rurali e rivieraschi, legati all'origine dei coloni da Pegli e dai suoi dintomi, non tali tuttavia da pregiudicare una piena intercomprensione, negli ultimi duecento anni, tra i Tabarchini e i locutori che fecero e fanno uso della koinè genovese: $\mathrm{cfr}$. in proposito Toso, in corso di stampa B. A dispetto della sua insularità totale, non a caso, Carloforte si dimostra più 
aperta alle innovazioni provenienti da Genova proprio in virtù dei maggiori contatti commerciali e mercantili, mentre la peninsulare Calasetta, accogliendo tali innovazioni in maniera più indiretta, conserva maggiormente i tratti rurali e rivieraschi, dimostrandosi più innovativa di Carloforte solo per quanto riguarda l'assunzione di sardismi.

Ben diverso è stato, significativamente, il destino dell'altra comunità nata dalla diaspora tabarchina, quella di Nueva Tabarca sull'llla Plana nei pressi di Alicante: il venir meno dei contatti con la madrepatria è certamente da annoverare tra le cause della totale scomparsa dall'uso, già all'inizio del sec. XX, della parlata tabarchina, completamente sostituita dallo spagnolo e dal catalano. Anche l'antica isola linguistica genovese di Bonifacio in Corsica (sec. XII), presso la quale il dialetto si dotò ben presto di un prestigio autonomo rispetto al genovese (Bonifacio godette di forme di autogoverno fino alla cessione della Corsica alla Francia, nel 1768) ha retto meno bene, alla lunga, al contatto linguistico col còrso, che è penetrato abbondantemente non solo nel lessico, ma anche nelle strutture fonetiche, morfologiche e lessicali. Per questi temi cfr. Toso, 2000a: 332-333, 340.

L'inventario toponomastico dei due comuni risulta bensì incrementato da un consistente apporto ligure, ma le forme indigene risultano numerose soprattutto a Calasetta, il cui territorio, a differenza di quello di Carloforte, era sfruttato dagli abitanti della vicina Sant'Antioco e del basso Sulcis per il pascolo e la raccolta di piante selvatiche. Denominazioni come Rio Tuppei, Regione Sisineddu, Mercureddu, Sa Scrocca Manna o la stessa Calasetta (Kädeséda in tabarchino, Kalezéra in sardo) ecc. denunciano la loro inequivocabile appartenenza a una stratificazione precedente all'installazione della colonia. È da rilevare ad ogni modo una discreta tendenza a genovesizzare i toponimi d'uso più frequente, come del resto avviene per le denominazioni di molti centri abitati esterni all'area di colonizzazione: ad esempio Igrệzi, Towlệa, Káǵe o Káǵay, Bậa Šteytíy sono rispettivamente, in tabarchino, i nomi di Iglesias, Teulada, Cagliari, Bosa e Stintino. Si coglie qui l'occasione per avvertire che la grafia utilizzata per le voci tabarchine è quella del Lessico Etimologico Italiano (LEI) diretto da Max Pfister.

20 Si veda ad esempio il caso di voci come ayǵọóni 'agnello', mulộni 'cippo di confine', muštayộni ‘spaventapasseri' ecc., nelle quali la vocale finale, dopo la nasale dentale, non tradisce il minimo sforzo di adattamento alla fonetica ligure.

21 Potrebbe essere, ad esempio, il caso di kaminẹéa 'sentiero lungo i bordi del campo, con caduta di - $r$ - intervocalica tipicamente ligure (la voce sarda originaria è kaminéra), o di kadín (< kaddínu). Significativamente, adeguamenti di questo tipo si rivelano più frequenti nei sardismi presenti a Carloforte o esclusivi di quella località.

22 VALLEBONA, 1988: 48: «La funzione di polizia e di vigilanza fu affidata dal Consiglio all'unica guardia municipale, il "mustassaffo" [...]. Il 10 gennaio 1740 il duca, da una terna di nomi presentatigli dal Consiglio, scelse come mustassaffo Antonio Danovaro, il quale prestò giuramento il successivo 10 marzo. Il 31 marzo Giovanni Cipollina, scelto dal duca come mustassaffo aggiunto, prestava giuramento nelle mani del Capitano di giustizia». La voce ha assunto ormai una connotazione prevalentemente scherzosa (v. oltre).

Cit. in Cabras - Rivano Poma, 1992 : 57. Il testo risale al 1844.

24 Su questo fenomeno in sardo sulcitano cfr. PIRAS, 1994: 105, 115-116, e VIRDIS, 1978: 19, 43.

25 Nostre informazioni.

26 In tabarchino è allofono del suono ligure «normale» (una $r$ leggermente meno vibrante di quella italiana), $\mathrm{e}$ lo si incontra nella pronuncia individuale, soprattutto delle persone al di sotto dei cinquant'anni, a Calasetta (BLASCO F:RRER, 1994: 188). Si potranno considerare d'influsso fonetico sardo forme isolate come tagęźzu 'pettegolo', in cui si riscontra un'ipercorrezione rispetto a lig. dagệzzu 'litigioso' (sec. XVIII dagaresso 'che le dà, che picchia'), la cui $d$ - fu sentita evidentemente come frutto della sonorizzazione della dentale iniziale in fonetica sintattica. Il fenomeno inverso si ha in dragẹ́tu 'traghetto', peraltro non generalizzato; più problematica la serie carlofortina ganbúza 'cambusa', garáfa 'caraffa', gardigún (lig. kardiǵún) 'carotide', gamẹ́lu 'cammello', gazệ́rma 'caserma', per la quale non mancano riscontri anche in area ligure.

27 «Per intrusione della sequenza sarda con cacuminale interna (lunga), si hanno anche prestiti con una dentale» (BLASCO FERRER, 1994: 188). Il tabarchino non limita peraltro la presenza di $-d$-intervocalico ai prestiti dal sardo e agli esiti regolari liguri dell' assimilazione del nesso romanzo -ld- ( $k a \dot{a} d a<$ CAL'DA): amplia il repertorio delle forme di tradizione semidotta o di provenienza straniera del tipo fursádu 'forzato', despētádu 'dispettoso', sprēžádu 'irriverente', ōzádu 'audace', manćinádu 'mancino', aǵáda 'salsa all'aglio', vergáda 'bastonatura', šakáda 'schiacciatura', assai diffuse già in genovese, con formazioni del tipo pasáda 'per- 
cosse', veptuláda 'colpo di vento', šï̌sáda 'soffiata', durmída 'dormita', fatigáda 'faticata', faćáda 'facciata' che non possono evidentemente attribuirsi a influsso sardo. Qui si pone anche il caso specifico di kapuná$d a$, un piatto tipico a base di galletta, tonno e verdure che si ritrova identico in Liguria, in Sardegna e presso le comunità tabarchine. L'origine catalana della voce non aiuta a chiarire l'area originaria di espansione del termine e del piatto stesso.

28 BLASCO FERRER, 1994: 189. L'estensione di $i$ al femminile plurale anche davanti a consonante ( $i$ škắrpe 'le scarpe') nella sottovarietà calasettana (ove è tuttaltro che generalizzata) e presso alcuni locutori carlofortini, si spiega più facilmente con un'estensione dell'analogo fenomeno che si riscontra in genovese davanti a vocale ( $i$ áe 'le ali', $i$ amíge 'le amiche') che con l'influsso della forma invariabile is del campidanese; lo stesso studioso rilevava del resto, giustamente (p. 176), la generalizzazione della neutralizzazione dell'articolo nell'accoppiamento con preposizione (ay štéle, day mugée) sia a Carloforte che a Calasetta.

29 Calasetta è a pochi chilometri da Sant'Antioco, linguisticamente sarda, con la quale si divide il territorio dell'isola omonima. Tuttavia, fino a tempi recenti, i contatti commerciali si svolgevano prevalentemente con Carloforte (vendita di prodotti agricoli, acquisto di generi vari, masserizie, articoli di lusso importati direttamente da Genova); per le antiche dispute legate alla divisione del territorio, tra Calasettani e Antiochesi vigeva una tradizionale rivalità, destinata a esplodere periodicamente durante le sagre paesane. Ciò spiega anche perché, come si vedrà più oltre, la manodopera avventizia impiegata nella viticoltura, venisse reclutata principalmente nel basso Sulcis e nella zona di Teulada.

30 Su 278 voci d'origine sarda inserite nella nostra selezione, 85 sembrano esclusive di Calasetta e 47 di Carloforte, ma tutte le altre sono comuni alle due comunità tabarchine, a volte con leggere varianti o con sfumature di significato. A Calasetta paiono prevalere voci legate a settori specialistici, soprattuto nel campo dell'agricoltura, a Carloforte sono più diffusi sardismi d'uso generale, oppure voci legate a un lessico di carattere espressivo. I sardismi nella varietà di Carloforte appaiono a volte meglio assimilati alla fonetica e alla morfologia del tabarchino, fatto che porta a ipotizzare stratificazioni diverse, e oggi difficilmente ricostruibili, lungo assi non solo diatopici, ma anche diacronici e diastratici. Una considerazione più approfondita di queste problematiche potrà essere affrontata dopo una più ampia sistemazione del lessico tabarchino nel progettato Dizionario Etimologico-Storico del quale si dirà più oltre.

31 Ciò a differenza dei veri e propri immigrati dal resto della Sardegna, che ancor oggi sentono immediatamente l'esigenza di apprendere il tabarchino per integrarsi pienamente nella realtà locale.

32 Cfr. nota 30. I lavoratori stagionali sardi provenivano per lo più dalla zona interna del basso Sulcis (Giba, Narcao, Piscinas, Masainas). Non sembra aver avuto conseguenze linguistiche la presenza di una frazione, Cussorgia, abitata da famiglie di lingua sarda: gli abitanti, generalmente mezzadri presso i proprietari calasettani, sono ed erano tutti bilingui.

33 «Iñ pọ́ 1 akapímu u sắrdu ma lẹ́ difísile $\mathrm{k}$ u se pẳrle» ('un po" lo capiamo il sardo, ma è difficile che qualcuno lo parli', informatrice calasettana di circa 50 anni).

34 'Nei primi tempi bisognava parlare loro in sardo, e per le parole che ignoravamo, ci facevamo capire... era più facile per noi parlare sardo, che per loro imparare il tabarchino... Abbiamo certe parole difficili, noi, fumaio$l o$, bue, cuore, non riescono a pronunciarle, abbiamo una parola che proprio non riescono a pronunciare, proprio difficile, fucile' (testimonianza di un agricoltore calasettano, classe 1912). Un informatore di circa sessantacinque anni sostiene: «Mi pắrlu u tabarkín e w sắrdu ánke, ší, mi sọ́ parlắ ánke w sắrdu e w sắrdu ke 1 ọ́ yparắu day karadwí de tộlệ́a kwánde veñívan ki pẹ̄ vendẹ́ñe» ('io parlo tabarchino e anche sardo, sì, so parlare anche il sardo che ho imparato dai carradori di Teulada quando venivano qui per la vendemmia').

35 Pẹ́gwa, šámu de pẹ́gwe. Allo stesso modo, è genovese la denominazione generica dei principali tipi di 'cesta' (kúfa, kaváñu), sarda quella legata a particolari specializzazioni (kartálu, kadín); è genovese il nome generico del 'truogolo' (árǵu < ALVEU), sardo quello di un tipo particolare di abbeveratoio in pietra, usato per i bovini (láku <LAQUEU), è genovese come si vedrà subito la denominazione della vigna, sarda quella relativa al suo impianto e alla sua coltivazione.

36 Cfr. ad es. sakắya 'agnella di un anno', semeytǘza 'pecora di un anno che non ha figliato'; qui rientra anche l'acclimatazione di angoộni 'agnello', forse facilitata dalla debolezza semantica della forma ligure bẹé, di origine onomatopeica.

37 La vulgata storica locale, appoggiandosi anche a una certa tradizione orale, attribuisce ai coloni piemontesi l'introduzione a Calasetta della viticoltura. In realtà il precario insediamento subalpino, oltre a non avere lasciato apprezzabili tracce linguistiche (di piemontesismi non comuni al sardo conosco soltanto la voce bregêéa 
'vagabonda', che potrebbe riprendere bargẹ́ra 'pastora': purché non si tratti di un francesismo importato dalla Tunisia) non sembra avere introdotto neppure pratiche vitivinicole specifiche: sia i vitigni (a partire dal principale, il carignano d'origine catalana), sia le tecniche di coltivazione e di produzione del vino rimandano inequivocabilmente al Campidano.

38 Si veda in proposito la ricerca di De Francesco - Leone, 1996.

39 «U peškắu de Kādesẹ́da u nu $\mathrm{l}$ ẹ štệtu mắi y peškáu adệšu, in peškău d amyá luntán. U kọ́pye sưlu. L ệa

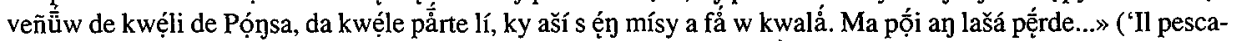
tore calasettano non è mai stato sveglio, un pescatore lungimirante. È solo capace a copiare. Quando arrivarono i Ponzesi, gente di quelle parti, anche qui cominciarono la pesca del corallo. Poi, hanno smesso...', informatore calasettano di sessant' anni). Le testimonianze orali sulla vita dei pescatori, sia a Calasetta che a Carloforte, si soffermano sulle dure condizioni di questa categoria, i cui membri erano costretti a lavorare a giornata presso i ricchi prupyetắi contadini o a compiere emigrazioni stagionali: «U peškắu w l ệa $\mathrm{\eta}$ mộ̂ttu dā

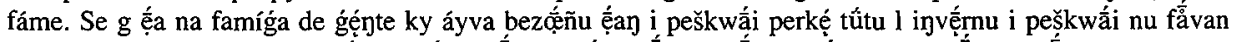

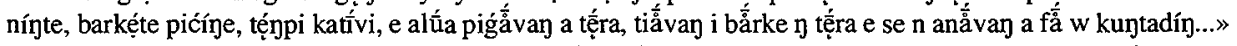
('Il pescatore era un morto di fame. Se c'era una famiglia bisognosa era quella del pescatore, non facevano nulla tutto l'inverno, per via delle barche piccole, del cattivo tempo, e allora stavano a terra, tiravano le barche a secco e facevano i giornalieri in campagna', informatore calasettano, classe 1912). La frequenza di cognomi siciliani (Sgrò, Ventagliò, Cincotti, Scopelliti) o ponzesi (Aversano, D'Amico) conferma la provenienza forestiera di molti pescatori, che sceglievano poi di trasferirsi presso le comunità tabarchine attratti dalla possibilità di partecipare stagionalmente alla tonnara o di arrivare a possedere un terreno. Ancor oggi a Calasetta si verifica una significativa migrazione stagionale di pescatori da Ustica e da Mazara del Vallo.

40 Tra i soprannomi raccolti a Carloforte e Calasetta numerosi, anche attribuiti a persone o famiglie di pretta origine tabarchina, utilizzano materiale lessicale sardo, scelto evidentemente in ragione di una particolare espressività, che si riconosce in genere nell'utilizzo di suffissi vezzeggiativi o peggiorativi: basti ricordare per

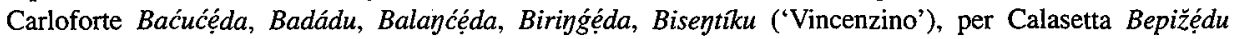
('Giuseppino'), Beližẹdu, Kazarẹ́du, Pulitẹ́du, ecc. Curioso è il nomignolo Pitanẹ́du attribuito a Carloforte alla statua del re Carlo Emanuele III, sul lungomare della cittadina.

41 L'utilizzo di suffissi d'origine sarda riguarda sia sostantivi d'uso comune (ćilẹ́da 'pene del bimbo', zigítu 'giocattolo', papelítu 'pezzetto di carta', forse veytižólu 'venticello', lebecóólu 'venticello di libeccio', rangitu 'zoppetto'), sia, soprattutto, i nomi propri e i loro ipocoristici, come Balíta 'Isabella', Bepika 'Giuseppina', Cícítu 'Francesco', Andrižín 'Andrea' ecc. Per completezza, va osservato che il suffisso -ọllu ha una discreta diffusione anche in Liguria, ove è comunque d'origine non locale. Quanto a -itu, è frequente in terraferma nei nomi propri importati dall'America Meridionale in seguito a consistenti fenomeni d'emigrazione di ritorno (Terezita, Markítu, Kwaysita 'Juancita', Kelítu 'Angelito'), e lo si incontra saltuariamente anche in sostantivi d'uso (papelítu). Sulla diffusione popolare della suffissazione d'origine spagnola in Liguria si veda Toso 1993.

Es. prevácu 'pretaccio', pwiáća 'grosso spavento'.

43 Tra le concordanze di natura fonetica o morfologica citeremo solo la desinenza del participio passato in -áu e la presenza ricorrente del fono $\breve{z}$. Tra i sardismi elencati da BLASCO FERRER, 1994: 189-190 per il tabarchino, l'occasionale somiglianza di forme lessicali liguri e sarde ha prodotto qualche interpretazione meritevole di approfondimento: ad esempio il tipo kú de sẹne 'colore di cenere' per 'grigio' è di larga diffusione in Liguria (cfr. PETRACCO SiCARDI - TOSO ET AL., d'ora in avanti VPL, s.v. curù) e non va quindi considerato, necessariamente, un calco sul campidanese kolóri (d)e žinížu. Lo stesso autore (p. 190) attribuisce a influsso sardo la voce tüydu 'piatto', attribuendola a specializzazione semantica dell'agg. sardo túndu 'rotondo', ma la voce è da sempre l'unica nota in Liguria in questo significato (VPL s.v.), ed è quindi arrivata in Sardegna coi Tabarchini. Anche un caso di concordanza casuale come bríska 'favo', presente in ligure (VPL s.v.) e in campidanese rende problematico l'accertamento dell'origine del tabarchino bríska, che appartiene peraltro a una sfera semantica interessata da vistosi fenomeni di prestito (v. Glossario). C'è da chiedersi se una voce come de báda 'gratis', di antica e diffusa presenza in Liguria, abbia visto rafforzata la sua vitalità in tabarchino grazie all'influsso del sardo de bbádas.

Oltre al caso già citato di bríška è particolarmente significativo, tra gli altri, quello di pwása 'potatoio': la voce compare identica in gran parte della Liguria, ma appartiene a un campo semantico, quello della viticoltura, nel quale il lessico tabarchino è in gran parte improntato al modello sardo. Un adattamento o sovrapposizione del campidanese pudása 'id.' non è quindi da escludere. Per il loro carattere di termini dell'uso generale non 
dovrebbero invece esservi dubbi sull' appartenenza al fondo originario del tabarchino di voci di antica e documentata presenza in Liguria come baysigå̀se 'dondolarsi', ćíkera 'tazza' (e ćikerúy), frazắ 'consumare', sẹ da búka 'palato', tián 'tegame', malgrado la corrispondenza riscontrabile coi tipi sardi bantsigái, cíkkera, fradzái, kẹlu dessa (b)úkka, tiánu.

45 Un caso tipico è quello di štánku 'tabaccaio', voce d'origine spagnola ben documentata in Sardegna (e pertanto indicata come sardismo in BLASCO FERRER, 1994: 189), ma altrettanto ben attestata in Liguria (Toso, 1993: 116); lo stesso si può dire per múñu 'crocchia' (in area ligure dal sec. XVII, Toso, 1993: 97) e per škabẹću 'modo di preparare il tonno', radicato sia in Liguria che in Sardegna (skabéčcu) e da connettere con sp. escabeche, cat. escabetx. Voci come trappáya e trappúzu tra le altre sembrano presupporre un tramite sardo (v. Glossario), ma non va dimenticato che l'ispanismo trampa è documentato in genovese del sec. XVII (Ibid., p. 121). Più complessa è la vicenda di baštášu 'facchino specializzato nel trasporto dei tonni nello stabilimento a terra': la voce è presente in sardo, ma la specializzazione semantica pare connetterla all'influsso lessicale siciliano, piuttosto consistente nel lessico della tonnara. In ogni caso la voce dovrebbe risalire, in ultima analisi, al cat. bastaix; tuttavia bastaxo si incontra anche in genovese del sec. XIII (ANONIMO GENOVESE, 1994: 549, rima 71, verso 34): l'esistenza di bastásu nel dialetto di Alassio (PEzzuolo, 1989: 17) renderebbe allora plausibile l'ipotesi di una sopravvivenza dell' antica voce ligure anche in tabarchino, se non fosse noto che da Alassio aveva luogo in passato una consistente migrazione stagionale di tonnarotti verso Carloforte e l'Isola Piana, da dove la voce sarà risalita sulla riviera ligure assieme a qualche altro termine tabarchino. Un esempio per tutti: la locuzione a ícu ícu 'appena appena', che corrisponde ad Alassio al tipo ligure comune a isa a ísa (PEzzuolo, 1989: 40), trova riscontro solo nel tabarchino a ícu ícu, che ha tutta l'aria di un «cavallo di ritorno» sulcitano in concorrenza col tipo genuinamente ligure (presente nella variante $a l$ ísu $a l$ isu). Altro caso interessante è quello dell'arabismo rắiže 'rais, capo della tonnara': il sardo arráis viene ascritto dal Wagner all'influsso siciliano, e ciò lascerebbe pensare che la voce tabarchina abbia seguito un analogo percorso: tuttavia proprio la forma ràixo è presente nel senso di 'capo di un equipaggio turco' in un testo genovese del 1781 (Toralbo Armonico in Toso, 2000b: 260), fatto che ne attesta la diffusione in area ligure indipendentemente dalla rete dalle relazioni sardo-sicule in cui i Tabarchini si trovarono coinvolti.

46 Sui francesismi in sardo cfr. DETTORI, 1988. Una voce tabarchina come geẹ́na 'imbarazzo' corrisponde sia a forme genovesi documentate già nel sec. XVIII, sia al sardo géńna 'noia, fastidio'.

47 WAGNER, 1997: 246-247 (d'ora in avanti: Ls. La classica opera del maestro della linguistica sarda, del 1950, verrà citata nel prosieguo sulla base di questa recente, ottima riedizione, con riferimento al numero delle pagine).

48 I miei informatori tabarchini sono in genere convinti, ad esempio, che la voce libán 'corda di sparto utilizzata in ambito marinaro' sia un prestito dal sardo su libánu, ma non sembra affatto improbabile il contrario, visto che libáy è voce genovese ben documentata nei vocabolari ottocenteschi: tale sembra essere anche l'opinione di WAGNER, 1960-64: II, 25 (d'ora in avanti, DES): «il punto d'irradiazione sarà stato Genova». Anche la voce māréla 'matassa di filo di foglia di palma nana' è presente in dialetto antiochese (nostre informazioni), ma non pare particolarmente diffusa in sardo: occorrerà verificare una eventuale derivazione della voce sarda dal tabarchino, visto che marẹ́la (VPL s.v.) è largamente attestato nella Liguria occidentale. Certamente c'è da chiedersi quale sia il rapporto tra lig. vašelẹ́a 'rastrelliera per i piatti' e il sardo antiochese fašelláu (con -ll-!) prima di ammettere che il tabarchino vašelá derivi dalla parlata sulcitana e non viceversa.

La voce malurédi 'gnocchi' segnalata per Carloforte da BLASCO FERRER, 1994: 190, è conosciuta (ma poco usata) con riferimento alla pasta di fabbricazione industriale, mentre per quella casalinga si continua a usare il termine ligure kasúli: essa pare quindi penetrata recentemente, attraverso l'italiano regionale. C'è da chiedersi se anche l'attuale (e, pare, piuttosto recente) diffusione dell' affermazione éya non sia stata veicolata dall'italiano regionale piuttosto che dall'adstrato sardo, e lo stesso potrebbe forse dirsi dell'uso enfatico di ǵa ('́a w sọ ke l ẹ́ tấrdi 'so bene che è tardi'), che, soltanto in quest'uso specifico, rappresenta comunque un elemento non assimilato rispetto al genuino $z a$. Riguardo alla fonetica, sembra da attribuire all'influsso dell'italiano regionale (o al sardo direttamente?) la propagginazione che si riscontra in voci più o meno recenti come lápizi 'lapis', grátizi 'gratis', tẹ́nnizi 'tennis': l'assimilazione dei forestierismi di questo tipo passa in ligure attraverso la caduta della $-s$ e l'allungamento di compenso della vocale precedente: $u$ lápi, agráti, $u$ tệni.

50 Il DEST ordinerà, commentandole, le voci raccolte a più riprese, nel corso di soggiorni variamente protrattisi nell' arco di dieci anni e attualmente ordinate in circa quindicimila schede. I materiali sono stati raccolti quasi esclusivamente attraverso la tecnica della conversazione libera, ma si è tenuto conto, a titolo di confronto, di fonti edite e inedite, come i materiali ALI raccolti da Ugo Pellis nel 1933, le voci calasettane inserite nel VPL (che peraltro espungeva programmaticamente i sardismi) e l'opera di VALLEBONA, 1987. 
51 SpANo, 1998 (riedizione dell'originale, Cagliari 1851; d'ora in avanti: Spano, con indicazione del volume in numeri romani e della pagina in cifre arabe).

52 WAGNER, 1996 (ripresa dell'originale Das ländliche Leben Sardiniens im Spiegel der Sprache, 1921); l'opera verrà d'ora in poi citata con la sigla Vr seguita dal numero di pagina dell'edizione italiana.

53 Le citazioni dal DES fanno seguire la sigla dall'indicazione del volume e, dopo la virgola, dal numero di pagina; quando l'apice di lemma sia una forma diversa da quella riportata, ciò viene indicato espressamente.

54 In linea di massima non sono stati riportati i derivati privi di reale autonomia semantica (es. žmamatüa $<$ $\check{z ̇ m a m a ́) ~ e ~ i n ~ p a r t i c o l a r e ~ q u e l l i ~ c h e, ~ p e r ~ e s s e r e ~ d i ~ c o n i o ~ l o c a l e, ~ a p p a r t e n g o n o ~ p i u t t o s t o ~ a l l a ~ s t o r i a ~ d e l ~ t a b a r c h i-~}$ no che a quella dei rapporti di tale parlata con il sistema sardo.

55 È stato naturalmente effettuato un controllo incrociato tra le attestazioni che in una prima fase risultavano esclusive delle due comunità: esso ha consentito di compiere interessanti osservazioni e correzioni; non si può tuttavia escludere che alcune delle voci presentate come esclusivamente calasettane o carlofortine siano in realtà conosciute, a vario livello, anche nell'altra comunità tabarchina.

56 Ringrazio la direzione dell'ALI e il dr. Giovanni Ronco per avermi consentito e agevolato la consultazione di questa fonte preziosa del lessico tabarchino.

57 In particolare, sono stati ridotti a $b, d$ e $g$ i tre segni sbarrati che indicano, rispettivamente, la fricativa bilabiale sonora, la fricativa dentale sonora e la fricativa velare sonora. Si avverte che le forme tabarchine registrate non tengono conto di varianti idiolettali (es. la pronuncia di $r$ polivibrante apicale in posizione intervocalica a Calasetta, la tendenza al passaggio $\breve{s}, \check{z}>s, z$ presso le giovani generazioni calasettane, la develar-

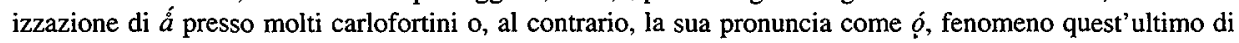
ampissima diffusione.

58 Come in sardo, il nome del 'corvo' (krốu), ad esempio, è passato a indicare anche il 'cormorano'.

59 Su questa voce cfr. anche le osservazioni di DeTTORI, 1993: 217, 222.

60 La variante potrebbe riflettere un influsso di lig. kumệ́lu 'un tipo, un tale' usato con funzione apotropaica per non citare il vero nome del rapace notturno, considerato apportatore di malasorte. In Liguria la 'civetta' ha infatti, accanto al nome vero, una serie di nomignoli e ipocoristici che evitano di fare ad essa riferimento diretto.

61 Per questa voce il Wagner (Vr206) risale ad alcune voci catalane; non escluderei comunque qualche collegamento con cirò 'nome di un vitigno calabrese coltivato nella zona di Cirò Marina'; in questo caso però, il TB potrebbe essere il tramite per la diffusione della voce in area campid.: in TB è infatti piuttosto frequente il

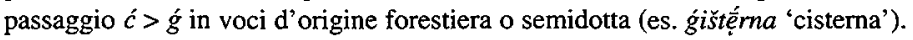

62 Cfr. anche il sardo regóttu Vr273 'ricotta': la concordanza potrebbe avere contribuito al mantenimento di rekệ́tu in luogo del sinonimo prešijs ș̣́a.

63 Di estremo interesse è lo slittamento semantico subito dal termine lig. originario, zú, passato ad indicare a Calasetta il 'pungolo per l'asino'. La voce è pressoché caduta in disuso.

64 «Erano per lo più braccianti i quali, esaurite le fatiche agresti ai loro paesi (e dopo la trebbiatura del grano, dell'orzo e dell'avena il lavoro di chi viveva nei campi, in Sardegna, si riduceva pressoché a zero), si muovevano alla volta di Calasetta come verso il Texas, il Colorado e l'Arizona puntavano le carovane dei cercatori d'oro. Una volta giunti, se s'erano già accordati con qualche contadino durante la stagione precedente (e tale impegno era sacrosanto per entrambi i contraenti) si recavano direttamente al magazzino del datore di lavoro, ove sostavano col carro in attesa che la vendemmia avesse inizio. Se l'accordo mancava, oppure era la prima volta che si raggiungeva Calasetta, secondo un'usanza invalsa nel tempo, i carri si schieravano l'uno dietro l'altro, come per una rassegna, sul Lungomare. Lì i carrolanti attendevano che qualche padrone di vigne stabilisse con loro un accordo, per lo più verbale e sancito da una stretta di mano, del valore di mille patti sottoscritti» (ROMBI, 1998: 88-89).

65 Un'altra denominazione familiare dell'asino, buríku, di probabile ascendenza spagnola, è presente sia in sardo (Spanol,285, Vr142) che in lig. (Toso, 1993: 61), fatto che rende problematico definime la provenienza in TB.

66 Di estremo interesse la distribuzione delle varianti lungo l'asse cronologico: l'informatore dell'ALI (trentaduenne nel 1933) proponeva il prestito non assimilato, una mia fonte ottantenne introduce la vocale di appoggio ma mantiene la -s- sorda, mentre l'uso attuale (informatore cinquantenne) presenta la sonorizzazione, sintomo di un definitivo adattamento del prestito al sistema TB. A CF si usa esclusivamente ápya (che è a sua 
volta un italianismo con adeguamento morfologico: il tipo lig., sconosciuto in TB, è á $v a$ ) o si confonde l'animale con la 'vespa'.

67 Qualche informatore propone anche kašẹ́ta di ábisi, di ápye 'cassetta delle api', che sembra un adeguamento semantico a partire da materiale lessicale lig.

68 La seconda variante denuncia un fenomeno tipico della sottovarietà carlofortina, ossia la confusione di -ñ-, $-n-$ e $-\eta$ - intervocaliche in un suono intermedio $-\tilde{y}$ - che viene spesso autocorretto dai parlanti per evitare omofonie fastidiose del tipo kaypáña 'campagna'/kaypáña 'campana'. Da qui forme ipercorrette come quella riscontrabile in kanína, ma anche, ad esempio, in vína 'vigna', che si sta diffondendo presso le giovani generazioni.

69 In realtà occorrerebbe verificare il significato del lig. ant. rixa, che ricorre in documenti quattrocenteschi, prima di escludere del tutto che la voce TB non rifletta piuttosto un arcaismo; né è da escludere che il termine sia penetrato dai dialetti it. merid. indipendentemente in TB e in sardo.

70 CABRAS, 1989, 185. Potrebbe appoggiarsi all'influsso del sardo kaltsọ́nes la fortuna di TB kāswín, voce certo non sconosciuta in area lig. ma assai meno diffusa del sinonimo brấge, che in TB è poco usato e considerato arcaizzante. Incerto è il caso della voce ǵipúy 'giubbone', ben documentato in area lig. ma corrispondente anche al sardo gippone (SpanoIl,59); depone a favore della sua appartenenza al fondo lig. del TB l'esistenza del dimin. gípunétu 'panciotto', anch'esso di antica e diffusa presenza in Liguria.

71 Unico caso tra le determinazioni di parentela, la voce sarda ha completamente sostituito altre forme affettive, e si è sostituita in pratica anche alla forma lig. non marcata affettivamente, pwẹ 'padre', che appare oggi di uso assai limitato, soprattutto a CA; analogamente, máma ha in pratica estromesso dall'uso mwẹ 'madre'.

72 «La processione si divideva in due gruppi: uno col povero Gesù trafitto sotto il baldacchino, e l'altro con la madre, la Madonna Maria, col velo nero sul capo, in cerca del figlio»: RoMBI, 1988: 57. Va avvertito che la cerimonia, di origine spagnola, tuttora praticata in Sardegna, fu ampiamente diffusa anche in Liguria durante il sec. XVII: gli encuentros di Genova e di Sanremo vengono paragonati dai viaggiatori dell'epoca a quelli fastosi di Milano e delle grandi città iberiche.

73 Andrebbe verificato un eventuale rapporto tra le voci sarde e la locuz. TB tiá de krúšte, lett. 'tirare la sfoglia', ma in senso fig. 'spettegolare'.

74 Nel caso di espressioni idiomatiche non assimilate è necessario mantenere una certa prudenza, in quanto alcune di esse potrebbero appoggiare la loro fortuna ad assonanze e concordanze lessicali col codice ricettore. Un caso per tutti è rappresentato dall'esclamazione tọka líku riscontrata da BLASCO FERRER, 1994: 159-160) in una registrazione fornitagli a Carloforte. Lo studioso la interpreta come sardismo: «Campidanese [tok:ái] è utilizzato come espressione di congedo o per interrompere una conversazione [...]; [lik:u] è un vezzeggiativo accorciato del nome di persona [sarbadorík:u]; [...] l'intera espressione può equivalere a: "e così basta!", "e non c'è bisogno d'altro"». Penso di poter proporre un'interpretazione non meno convincente alla luce di una interiezione del tutto analoga che si ritrova in un testo genovese del 1746: «Ro convòio o l'è chì vexin,l o s'è partìo da Portofin,/n'à portao d'ògni mercanzìa:/tocca e lecca e marcia via» (in GaLLıNO, 1997: 48): mi sembra probabile che l'espressione sarda si sia quanto sovrapposta in qualche modo su quella ligure. Analogamente offro una variante all'interpretazione di un altro modismo proposta da BLASCO FERRER, 1994: 160: in avyẹ́mu da reštá nứi e krû́i kúme l ō̌éllu du bẹ́ca lọka 'rimarremo nudi e crudi come l'uccello di beccialo-

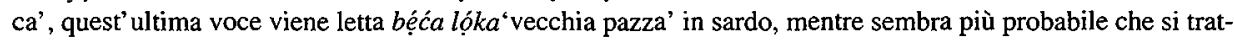
ti del genovese béća l óka 'colui che fotte le oche', sia tenendo conto del significato osceno di 'uccello', sia del fatto che il personaggio in questione è comunque di sesso maschile (del beccialoca).

75 Alludo non solo a miti ricorrenti nella pubblicistica locale (e persino in luoghi comuni diffusi a livello popolare), come quello dell' «operosità» ligure che sarebbe alla base delle fortune economiche delle due comunità; ma anche a elementi specifici della cultura materiale, a partire dall'introduzione negli ultimi decenni di piatti tipici liguri nella cucina carlofortina, forse anche nell'intento di enfatizzare e valorizzare a scopi turistici la «diversità» tabarchina rispetto alla Sardegna, in alcuni aspetti particolarmente attraenti per chi arrivi dall'esterno. Sotto questo aspetto Calasetta si dimostra meno influenzata dal rapporto col «centro» genovese. Lo dimostra ad esempio lo stesso arredo urbano, ancora fedele al modello originario importato dalla Tunisia (le case bianche a un solo piano), rispetto alla trasformazione subita da Carloforte, adeguatasi ai colori tipici della Riviera, al punto da essere paragonata ad Alassio o a Santa Margherita Ligure sulle guide turistiche. Che si tratti di un fenomeno relativamente recente lo dimostra la descrizione che BotTIGLIONI, 1928: 2, fa della cittadina tabarchina parlando delle «sue casette tutte bianche, raggruppate verso il porto»: un quadretto ormai del tutto irriconoscibile nell'odierna Carloforte, che si adatterebbe invece, semmai, all'altra comunità. 
'Se vado per mare i Turchi mi catturano, se vado per terra i Sardi mi ammazzano'.

77 Dopo la sua fondazione, Carloforte fu razziata due volte dai pirati tunisini, e gran parte della popolazione fu ridotta in temporanea schiavitù. Una schiava tabarchina, Francesca Rosso, andò poi sposa al bey di Tunisi e fu madre di Ahmed il Sardo, sovrano aperto, nella prima metà del sec. XIX, all'influsso dell'Occidente anche grazie ai buoni uffici del suo primo ministro, l'imprenditore d'origine genovese Giovan Battista Raffo. Alla luce della stretta interrelazione economica tra le comunità tabarchine e il retroterra sulcitano, appare evidente il carattere enfatico del proverbio, usato essenzialmente per indicare una situazione di incertezza, e la cui funzione precipua sembra essere quella di riassumere l'originalità dell'esperienza tabarchina, molto al di là del suo significato letterale.

78 In questo caso, non sembrano sussistere rapporti di ostilità o prevenzioni di alcun genere, e l'integrazione, perseguita in genere dal nuovo venuto, diventa assoluta: ho informatori con cognomi tipicamente sulcitani, figli di immigrati recenti, che definiscono orgogliosamente se stessi Tabarchini; lo stesso vale, come si è visto, per persone provenienti da altre regioni.

79 L'uso corrente del diminutivo sardẹtu, sardọtu ha in generale una sfumatura ironica ma non apertamente spregiativa, legata anche alle effettive caratteristiche fisiche dei Maureddus - i Sardi del Sulcis - normalmente più bassi di statura rispetto ai Tabarchini.

80 È sintomatico sotto questo punto di vista come il rapporto, pur storicamente difficile, tra i Calasettani e i vicini abitanti della sarda Sant'Antioco venga oggi risolto in forme non troppo dissimili dai consueti blasoni popolari che contraddistinguono anche comunità vicine non caratterizzate da alterità di tipo etnico-linguistico: gli antyogấiži, sono definiti semplicemente barộži 'seccatori, millantatori' o anche grūtááiži 'cavernicoli' per la presenza fino a tempi recenti, nella cittadina sulcitana, di dimore ipogee (questa voce è un calco del sardo grotáyus con apposizione del solito suffisso-ENSE). Gli Antiochesi definiscono a loro volta i Calasettani tabakinus forazántus 'Tabarchini ladri di santi' in ricordo della sottrazione, avvenuta in epoca imprecisata, di una effigie della Madonna della Misericordia da una chiesetta che sorgeva in un territorio conteso tra le due comunità. Cfr. ancora CABRAS, 1989: 184: «Si camminava di gran lena fino alle prime case del centro abitato dove restavamo più uniti per paura che i ragazzi di Sant'Antioco ci sfottessero per attaccar briga. Si verificava di sovente che dicessero ai Calasettani: "Tabakinu tabakineddu iscua e scueddu, iscua e molenti", che non so neppure tradurre ma che in sostanza significava che chi parlava il tabarkino era un asino [...]. Qualche volta da lontano giungevano sassate e noi via a correre ripromettendoci una bella vendetta allorché fossero giunti a Calasetta in occasione della festa di Santa Lucia, giacché questo era l'uso per l'accorrere di antiochensi alla ricorrenza del tredici dicembre allorché da noi si faceva gran festa».

81 'Pensi che il sardo abbia lasciato qualche traccia nel tabarchino?'. 'Niente; quasi niente; cioè, ci sono parole, nella nostra lingua, che certamente sono d'origine sarda... sul momento non me ne vengono in mente... si tratta di espressioni, non so, di esclamazioni...'.

82 CABRAS, 1993: 6-7.

83 Vi figurano tra l'altro aîsku, kazára, paraštáǵu, trabúsu. Analogamente, un'appendice di «Parole da sálvare» pubblicata in SIMEONE, 1992: 107-113, accoglie volentieri voci di provenienza sarda.

84 Tipico il commento «ma a nu l ẹ́ pắula nọ́štra» 'non è una nostra parola' o «šta ki l ẹmu piǵá da lú» 'questa l'abbiamo presa da loro (dai Sardi)', che accompagna il commento di una voce d'origine sarda.

85 «A I ẹ́ na páula da víña» 'è una parola legata alla campagna', «Se ge díže kuší tántu pe ríe» 'è un termine dell' uso scherzoso'. Nel controllo incrociato tra i materiali di Carloforte e quelli raccolti a Calasetta, gli informatori commentavano i sardismi esclusivi dell'altra comunità tabarchina con espressioni del tipo «é, šta ki ā kunúšu ma da nyắtri a nu se dớvye» 'sì, è una parola che conosco, ma noi non la usiamo', «ẹ́n páwín sắrde $\mathrm{k}$ á piǵá y kādesedệi» 'sono parole sarde in uso a Calasetta', e così via.

\section{Bibliografia}

ALI = Atlante Linguistico Italiano (materiali).

Anonimo Genovese (1994), Rime e ritmi latini, ed. a cura di J. NiCOLAS, Commissione per i testi di lingua, Bologna.

Blasco Ferrer, E. (1994), Contributo alla conoscenza del ligure insulare. Il tabarchino di Sardegna, «Zeitschrift für romanische Philologie», 110, 1/2, pp. 153-194. 
Bottiglioni, G. (1928), L'antico genovese e le isole linguistiche sardo-corse, «L'Italia Dialettale», IV, pp. 1-76.

Cabras, G. (1989), Calasetta anni Venti, in proprio, Roma.

Cabras, G. (1993), Calasetta. Difesa di un dialetto. Seconda edizione aggiornata, TEA, Cagliari.

Cabras, M - Rivano Poma, P. (1992), Calasetta. Storia e tradizione orale, TEA, Cagliari (Iled.).

De Francesco, M. - Leone, A. (1996), Gente di mare. Vicende e personaggi della marineria dell'Isola di San Pietro, E. Gasperini Editore, Cagliari.

DES = Wagner, M.L. (1960-1964).

DEST $=$ Toso, F., Dizionario Etimologico-Storico Tabarchino (materiali).

Dettori, A. (1978-80), La collaborazione dello Spano alle traduzioni bibliche di L. L. Bonaparte, «Studi Sardi», XXV, 1978-80, pp. 285-335.

Dettori, A. (1988), Francesismi nel dialetto di Cagliari, «Annali della Facoltà di Lettere e Filosofia dell'Università di Cagliari», N.s., VIII, pp. 277-305.

Dettori, A. (1993), Lineamenti di omitonimia sarda, «Quaderni di Semantica», 14, 2, pp. 211-247.

Fusco, F. - Orioles, V. - Parmeggiani, A. (2000), Processi di convergenza e differenziazione nelle lingue dell'Europa medievale e moderna, Forum, Udine.

Gallino, G. (1997), Cadenna zeneise e poesie anonime sulla guerra del 1746-1747 a c. di F. Toso, Le Mani, Recco.

LEI = Pfister, M. (dal 1971).

Ls = Wagner, M.L. (1997).

Orioles, V. (1997), Saggio introduttivo a B. Di Pietro, Ami d'carätter (Uomini di carattere). Racconti nel dialetto galloitalico di San Fratello (Messina), Furci Siculo.

Orioles, V. - Toso, F. (in corso di stampa), Insularità linguistica e culturale. Documenti del Convegno Internazionale di Studi (Calasetta, 23-24 settembre 2000).

Petracco Sicardi, G. - Toso, F. et Al. (1985-1992), Vocabolario delle Parlate Liguri, Consulta Ligure, Genova (4 voll.).

Pezzuolo, S.B. (1989), Dizionario Alassino, Stalla, Albenga.

Pfister, M. (dal 1971), Lessico Etimologico Italiano, L. Reichert Verlag, Wiesbaden.

Piras, M. (1994), La varietà linguistica del Sulcis. Fonologia e morfologia, Edizioni della Torre, Cagliari.

Rombi, B. (1988), Un anno a Calasetta, Ecig, Genova.

Simeone, N. (1992), Grammatica tabarkina, Bandecchi e Vivaldi, Pontedera.

Sitzia, P. (1998), Le comunità tabarchine della Sardegna meridionale: un'indagine sociolinguistica, Condaghes, Cagliari.

Sobrero, A. (1969), Alcuni fenomeni di disgregazione recentemente osservati nel tabarchino, «Bollettino della Carta dei Dialetti Italiani», IV, pp. 187-196.

Sobrero, A. (1971), Fenomeni di disgregazione recentemente osservati nel tabarchino, «Parole e Metodi. Bollettino dell'Atlante Linguistico Italiano", I, pp. 1-11.

Sobrero, A. (1974), Il tabarchino: processi di disgregazione linguistica in atto, in Id., Dialetti diversi. Proposte per lo studio delle parlate alloglotte in Italia, Milella, Lecce 1974, pp. 17-32.

Spano $=$ Spano, G. (1998) .

Spano, G. (1998), Vocabolariu sardu-italianu, a cura di G. Paulis, Ilisso, Nuoro (IIed., 2 voll.)

Toso, F. (1993), Gli ispanismi nei dialetti liguri, Ed. dell'Orso, Alessandria.

Toso, F. (1999), La componente ligure nel lessico capraiese, «Zeitschrift für romanische Philologie», 115, 3, pp. $472-501$.

Toso, F. (2000a), Per una storia linguistica del genovese d'Otramar, in Fusco-Orioles-Parmeggiani (2000), pp. 327-341.

Toso, F. (2000b), La letteratura in genovese, vol. II, L'età repubblicana, Le Mani, Recco.

Toso, F. (in corso di stampa A), Specificità linguistica e percezione dell'altro nella società tabarchina contemporanea, in Atti del Convegno Internazionale di Studi «Che cosa ne pensa oggi Chiaffredo Roux?» Percorsi della dialettologia percettiva all'alba del nuovo millennio (Bardonecchia 25-27 maggio 2000).

Toso, F. (in corso di stampa B), Conservazione e innovazione in tabarchino, in Atti del Convegno Internazionale di Studi Una lingua del mare: il genovese tra Liguria e Mediterraneo (Genova-Arenzano, 22-23 novembre 1998).

Tropea, G. (1970), La letteralizzazione dei dialetti galloitalici di Sicilia, "Atti del Convegno di Studi su lingua parlata e lingua scritta", Bollettino del Centro di Studi Filologici e Linguistici Siciliani, 11, pp. 3-31.

Vallebona, G. (1987), Dizionario Tabarkino-Italiano, Compagnia dei Librai, Genova.

Vallebona, G. (1988), Carloforte. Storia di una colonizzazione, Edizioni della Torre, Cagliari (IIIed.).

Virdis, M. (1978), Fonetica del dialetto sardo campidanese, Edizioni della Torre, Cagliari. 
$\mathbf{V r}=$ Wagner, M.L. (1996).

VPL $=$ Petracco Sicardi, G. - Toso, F. et Al. (1985-1992).

Wagner, M.L. (1960-1964), Dizionario etimologico sardo, Winter, Heidelberg.

Wagner, M.L. (1996), La vita rustica della Sardegna riflessa nella lingua. Saggio introduttivo, traduzione e cura di G. Paulis, Ilisso, Nuoro (Iled.).

Wagner, M.L. (1997), La lingua sarda. Storia, spirito e forma, a c. di Giulio Paulis, Ilisso, Nuoro (IIed.).

Povzetek

\section{SARDSKE JEZIKOVNE PRVINE V LIGURSKEM GOVORU TABARKINOV}

Prispevek skuša osvetliti govor, imenovan tabarchino; to jezikovno enoto tvorijo prebivalci dveh naselij, Carloforte in Calasetta, na otokih San Pietro in San Antioco ob jugozahodni obali Sardinije: koloni, ki so poprej bivali v Tuniziji kot ostanki genoveške kolonizacije iz 16. stoletja, in sicer na otoku Tabarca, odtod ime govora, so v 18. stoletju prinesli s seboj ligursko-genoveški govor. Ohranjanje originalnega govora kaže $z$ ene strani kolektivno sposobnost prebivalstva, da se obdrži jasna razlika s sardščino na otoku, $z$ druge pa socioekonomski prestiž tabarkinske skupnosti in pa stalne vezi $\mathrm{z}$ matično deželo in te so utrjevale genoveško narečje. Kljub temu, da so bili Tabarkini docela v sardskem okolju, se je njihov val obnašal kot vsi kolonialisti: izrabljanje in izvažanje krajevnih virov za življenje. Ta dejavnost pa obenem v dobri meri utemeljuje iz sardščine prevzete besede in tudi potek prevzemanja: čeprav jih je veliko in kažejo, da je prišlo do prevzemanja že v prvi dobi naseljevanja, so prevzete besede $\mathrm{v}$ glavnem omejene na agrarno življenje in nanj vezano dejavnost; ali pa kažejo posebnosti okolja (krajevna imena, oblike tal, rastlinstvo), torej to, česar doseljenci niso mogli prinesti s seboj. Sardsko besedje je kdaj pa kdaj pogojeno z močno izraznostjo, vendar ni prevladalo nad ligursko komponento v govoru Tabarkinov v vseh tistih situacijah, ki so zahtevale jezikovno skladnost z genoveškim narečjem, torej povsod tam, kjer posebnih razlogov ni bilo, kjer je tradicija obstala.

Študija obravnava v svojem prvem delu nekaj splošnoveljavnih ugotovitev o jezikovnih posledicah stičnosti dveh jezikovnih variant; ugotavlja pa, da mogočno število sardizmov ni načelo glasoslovno in oblikovno-skladenjsko podobo tabarkinskega govora: izposojenke iz sardščine so se vanj vklopile, ne da bi povzročile kakršno koli nelagodnost v fonološkem sistemu ali v skladenjskih strukturah. Sledi izbran seznam izrazov, ki so sardskega izvora, z etimologijo in semantičnimi opombami, kjer je bilo to potrebno. Končni del študije je namenjen podrobni obravnavi sociolingvističnih problemov, ki se porajajo, ko tabarkinsko govoreči prebivalci sprejemajo izraze iz sardščine. K temu se še dodaja, da sta obe jezikovni skupnosti, tista $v$ Carloforte in tista $v$ Calasetti, na različen način izpostavljeni tujim jezikovnim vplivom - misli se na italijanščno, uradni jezik in jezik v javni rabi in da prihaja $\mathrm{v}$ besedju celo do neke težnje $\mathrm{k}$ purizmu. 